OPEN ACCESS

Edited by:

Xiaogang $W u$,

Institute for Systems Biology, United States

Reviewed by: Farah Sheikh, University of California, San Diego, United States

Ju Chen,

University of California, San Diego, United States

*Correspondence: Xuejun Wang

xujeun.wang@usd.edu

${ }^{\dagger}$ Present Address: Travis Bjordahl, Bierschbach Dental, Milbank, SD, United States

Specialty section: This article was submitted to Systems Biology, a section of the journal Frontiers in Physiology

Received: 14 June 2017

Accepted: 31 July 2017 Published: 17 August 2017

Citation: Abdullah A, Eyster KM, Bjordahl T, Xiao P, Zeng E and Wang X (2017) Murine Myocardial Transcriptome Analysis Reveals a Critical Role of COPS8 in the Gene Expression of

Cullin-RING Ligase Substrate Receptors and Redox and Vesicle Trafficking Pathways. Front. Physiol. 8:594.

doi: 10.3389/fphys.2017.00594

\section{Murine Myocardial Transcriptome Analysis Reveals a Critical Role of COPS8 in the Gene Expression of Cullin-RING Ligase Substrate Receptors and Redox and Vesicle Trafficking Pathways}

\author{
Ammara Abdullah ${ }^{1}$, Kathleen M. Eyster ${ }^{1}$, Travis Bjordahl ${ }^{1+}$, Peng Xiao ${ }^{1}$, Erliang Zeng ${ }^{1,2}$ \\ and Xuejun Wang ${ }^{1 *}$ \\ 1 Division of Basic Biomedical Sciences, Sanford School of Medicine of the University of South Dakota, Vermillion, SD, \\ United States, ${ }^{2}$ Department of Computer Science and Department of Biology, University of South Dakota, Vermillion, SD, \\ United States
}

Background: The COP9 signalosome (CSN) consisting of 8 unique protein subunits (COPS1 through COPS8) serves as the cullin deneddylase, regulating the catalytic dynamics of cullin RING ligases (CRLs), the largest family of ubiquitin ligases. Supported primarily by the decrease of substrate receptor (SR) proteins of CRLs in cells deficient of a CSN subunit, CSN-mediated cullin deneddylation is believed to prevent autoubiquitination and self-destruction of the SR in active CRLs. However, it is unclear whether the decrease in SRs is solely due to protein destabilization. Moreover, our prior studies have demonstrated that cardiac specific knockout of Cops8 (Cops8-CKO) impairs autophagosome maturation and causes massive necrosis in cardiomyocytes but the underlying mechanism remains poorly understood. Given that Cops8 is nucleus-enriched and a prior report showed its binding to the promoter of several genes and association of its ablation with decreased mRNA levels of these genes, we sought to determine the dynamic changes of myocardial transcriptome in mice with perinatal Cops8-CKO and to explore their functional implications.

Methods and Results: Myocardial transcriptomes of Cops $8^{\text {flox/flox }}$, Cops $8^{\text {flox } /+}::$ Myh6-Cre, and Cops8flox/flox ::Myh6-Cre littermate mice at postnatal 2 and 3 weeks were analyzed. The data were imported into an in-house analysis pipeline using Bioconductor for quantile normalization and statistical analysis. Differentially expressed genes (DEGs) between groups at each time point or between time points within the group were revealed by $t$-test. Genes with $p<0.05$ after Benjamini and Hochberg false discovery rate correction for multiple hypothesis testing were considered as significant DEGs. We found that (1) the Ingenuity Pathway Analysis (IPA) revealed significant enrichment of DEGs in multiple pathways, especially those responding to oxidative stress, in homozygous Cops8-CKO hearts at both 2 and 3 weeks, corroborating the occurrence of massive cardiomyocyte necrosis at 3 weeks; (2) the 
decreases in multiple CRL SR proteins were associated with decreased transcript levels; and (3) enrichment of DEGs in the chromatin remodeling pathway and the microtubule motility and vesicle trafficking pathways.

Conclusions: Our data are consistent with the notion that Cops8/CSN plays a role in the transcriptional regulation of $C R L S R s$ and in the redox and vesicle trafficking pathways.

Keywords: the COP9 signalosome, transcription profiling, myocardium, COPS8 gene, conditional gene targeting, cullin-RING ligases, F-box proteins, mouse

\section{INTRODUCTION}

Originally identified in mutant Arabidopsis that exhibits constitutive photomorphogenesis (Wei and Deng, 1992), the COP9 signalosome (CSN) is evolutionally conserved from yeast to humans, with its bona fide biochemical activity being cullin deneddylation responsible for the deconjugation of the ubiquitinlike protein NEDD8 from the cullin scaffold subunit of cullinRING ligases (CRLs) (Lyapina et al., 2001; Bosu and Kipreos, 2008; Sozen et al., 2015). The assembly of a CRL is centered on a cullin scaffold which binds a substrate receptor (SR) module at its amino terminal end and a catalytic RING-containing protein (either RBX1 or RBX2) at its carboxyl terminal end. Covalent attachment of NEDD8 to the cullin via a ubiquitination-like process known as neddylation activates the CRL while CSNmediated cullin deneddylation is also required for the proper functioning of most, if not all, CRLs (Wei and Deng, 2003; Chamovitz, 2009). CRLs are the largest family of ubiquitin E3 ligases; hence, the CSN plays an important role in regulation of ubiquitination. In higher eukaryotes, the CSN holocomplex consists of 8 unique protein subunits (CSN1 through CSN8) (Wei and Deng, 1999) where the deneddylase enzymatic activity resides in the metalloenzymatic motif or JAMM domain of CSN5 subunit (Cope et al., 2002). CSN1-CSN4, CSN7, and CSN8 are the six PCI (Proteasome lid-CSN-Initiation factor 3) domain-containing proteins whereas CSN5 and CSN6 are the two MPN (MPR1/PAD1 amino-terminal) domain-containing proteins. The crystal structure of the CSN holoenzyme reveals that the 6 PCI's form a horseshoe-shaped ring where $\alpha$-helices of all subunits form a large bundle. CSN5 and CSN6 are present at the core of this bundle. In the absence of substrate, CSN5 is autoinhibited. CSN4 senses the binding of neddylated CRL whereas CSN5 with the help of CSN6, specifically removes NEDD8 from CRLs (Lingaraju et al., 2014). Binding of either the CSN or a CRL substrate to a neddylated CRL is a competitive mechanism. According to the current model, when the substrate for an active CRL runs low, the CSN binding and subsequent deneddylation of CRLs prevents the autoubiquitination and resultant destruction of the SR proteins of the CRL complex (Wang and Martin, 2015). On the other hand, binding of substrate to CRL complexes markedly inhibits deneddylation by the CSN. In fact, binding of substrate to the CSN-CRL complex facilitates dissociation of the CSN (Emberley et al., 2012). Dissociation of the CSN from the unloaded and deneddylated CRL allows binding by CAND1 (Cullin-Associated and Neddylation-Dissociated 1) protein. The CAND1 dissociates the old SR module for a new SR, which allows formation of a new CRL that is specific for a different class of substrates. The preserved SRs can also complex with the cullin and RBX-assembly of no longer needed CRLs and maintain homeostasis of CRL substrates in the cell (Schmidt et al., 2009; Pierce et al., 2013; Wang and Martin, 2015). Given the indispensable role of the CSN in propelling cell division cycle progression and sustaining cell survival, targeting the CSN for treatment of cancer has long been sought and, with the identification of small molecular inhibitors of CSN5 (Schlierf et al., 2016), this strategy has shown great promise; hence, it becomes more significant to have a better understanding of the impact of CSN impairment on the wellbeing of vital organs, such as the heart.

Ablation of any one of the CSN subunits leads to loss of the holocomplex and cullin deneddylation activity (Busch et al., 2007). Therefore, the entire CSN holocomplex is required for cullin deneddylation although the deneddylase activity of the CSN only resides in CSN5 (Wei and Deng, 2003). The CSN8, the smallest and the least conserved CSN subunit, is encoded by the COPS 8 gene. We have previously reported that CSN8 is required for the deneddylation of cullins in cardiomyocytes and that a defect in deneddylation activity due to conditional knockout of the Cops8 gene in cardiomyocytes (CSN8-CKO) leads to decreased expression of SR proteins, such as Fbxo32, VHL and Fbxw1a, in the heart (Su et al., 2011b). Our group has also provided compelling evidence that CSN8/CSN regulates not only the ubiquitin-proteasome system (UPS) but also the autophagic-lysosomal pathway for cardiac protein quality control and that CSN8-CKO initiated in perinatal and adult mice causes massive cardiomyocyte necrosis and heart failure ( $\mathrm{Su}$ et al., 2011a,b, 2013). However, the molecular basis underlying these CSN8/CSN functions in the heart remains poorly understood.

Most studies have defined CSN8/CSN as a post-translational regulator of protein degradation. CSN8 function at the transcriptional level has rarely been investigated in vertebrates although the CSN was originally identified as a transcriptional repressor (Wei and Deng, 1992; Wei et al., 1994). CSN subunits have been evaluated for their role beyond the regulation of protein stability and it was suggested that CSN subunits might have a direct role in the regulation of gene expression (Wei et al., 2008; Chamovitz, 2009). In human HEK293 cells, silencing of CSN5- caused a decrease in F-box protein 4 (Fbx4) expression (Cope and Deshaies, 2006). In mice, CSN5-deleted thymocytes showed decreased expression of $I \kappa B-\alpha$ mRNA (Panattoni et al., 2008). Similarly, CSN8 conditional knockout in peripheral $\mathrm{T}$ cells caused aberrant gene expression of several cell cycle genes 
and chromatin immunoprecipitation assays detected CSN8 at the promoter regions of these genes (Menon et al., 2007). We have previously shown that CSN8 is enriched in the nuclei of cardiomyocytes (Su et al., 2011b), consistent with a role of CSN8 in regulation of cardiac gene expression. However, the role of CSN8 as a potential transcriptional regulator in the heart has not been tested. We therefore sought to determine the impact of cardiac CSN8 deficiency on myocardial gene expression. The impact of loss of a CSN subunit on the full transcriptome of an organ in a vertebrate animal has not been described although the gene expression profile of ablation of CSN subunits, such as CSN4 and CSN5 in lower species (e.g., Drosophila) were reported (Oron et al., 2007).

Using whole-genome DNA microarray, here we profiled the dynamic changes in myocardial transcriptome of mice with perinatal CSN8-CKO. Our analyses demonstrate for the first time that CSN8-CKO has a major effect on the transcript levels of genes enriched in multiple pathways including signaling pathways in responses to oxidative stress, microtubule dynamics and vesicle trafficking, corroborating very well with the functional changes in the heart. Further verification of the expression changes at the protein level reveals that the reduced protein levels of many SR proteins of CRLs in myocardium with CSN8-CKO are associated with decreased expression at the transcript level, suggesting that CSN8/CSN plays an important role in promoting the gene expression of the SR in addition to protecting them from autoubiquitination.

\section{MATERIALS AND METHODS}

\section{Mice}

We have previously described generation of CSN8-CKO mice with the use of the Cre-loxP system in which transgenic Cre expression is driven by the mouse $\alpha$-myosin heavy chain (Myh6) promoter (Su et al., 2011b). The Cops $8^{\text {flox/flox }}$ (CTL) and Cops $8^{f l o x /+}::$ Myh6-Cre (Heterozygous Cops8 knockout or Het-CKO) littermates of Cops $8^{\text {flox/flox }:: M y h 6-C r e ~(h o m o z y g o u s ~}$ CSN8-CKO or Hom-CKO) mice were used. The knockout of the Cops8 gene which encodes CSN8 was confirmed with real time reverse transcription PCR ( $\mathrm{RT}^{2}$-PCR) where primers for the $5^{\prime}$ UTR region and exon 5 of the Cops 8 gene were used (Supplementary Figures 1A,B). This study was carried out in accordance with the recommendations of the Guide for the Care and Use of Laboratory Animals (US Department of Health, Education, and Welfare, Department of Health and Human Services, NIH Publication 85-23). The protocol for care and use of animals in this study was approved by the Institutional Animal Care and Use Committee of the University of South Dakota.

\section{RNA Extraction and Purification}

Total RNA from mouse ventricular myocardial samples was extracted and purified using the RNeasy plus mini kit (Catalog number: 74134) from Qiagen. Briefly, 30-50 mg of ventricular myocardial tissue was homogenized in $1 \mathrm{~mL}$ of TRI reagent with a Polytron homogenizer. Bromochloropropane $(200 \mu \mathrm{L})$ and $3 \mathrm{M}$ sodium acetate $(60 \mu \mathrm{L})$ were added and the homogenate was centrifuged. After centrifugation the aqueous layer was collected, mixed with RLT buffer (Qiagen, Valencia, CA) and ethanol, and centrifuged through a Qiagen RNeasy column. The column was washed and treated with ribonuclease-free deoxyribonuclease (Qiagen) to remove any potentially contaminating DNA. The total RNA was then eluted from the column.

\section{RNA Quality Control and Quantification}

RNA samples were tested for their quality and quantity by microfluidics analysis using a RNA 6000 Nano LabChip kit in an Agilent Bioanalyzer (Agilent Technologies).

\section{Microarray Gene Expression Analysis}

Gene expression in the myocardium of CTL, Het-CKO, and Hom-CKO littermate mice at 2 and 3 weeks of age $(n=3$ mice/group, both sexes were used for each group) was analyzed using DNA microarrays (CodeLink Whole Mouse Genome Bioarrays, Applied Microarrays, Inc., Tempe, AZ) as described previously (Eyster et al., 2007). Briefly, the MessageAmp II aRNA Amplification kit (Ambion/ThermoFisher) was used for the synthesis of biotinylated cRNA. RNA in the samples was reverse transcribed into first strand complementary DNA (cDNA) and the second strand of cDNA was then synthesized. Complementary RNA (cRNA) was synthesized from the doublestranded cDNA in a reaction that incorporates biotin-11-UTP. The biotinylated cRNA was purified on a Qiagen RNeasy column and fragmented. The fragmented cRNA was hybridized with the DNA microarrays for $18 \mathrm{~h}$ at $37^{\circ} \mathrm{C}$. The slides were washed and incubated with streptavidin-Alexa Fluor 647 (Molecular Probes) and washed again. The slides were scanned with an Axon GenePix 4,000B Scanner. Gene expression values were obtained from a summarization of intensity values of all corresponding probes using the RMA (Robust Multi-array Average) method. The pre-processed microarray data were imported into an in-house analysis pipeline using Bioconductor for quantile normalization and statistical analysis.

The choice of the 2- and 3-week-of-age time points was based on the phenotype of these mice. Overt left heart failure as reflected by increased lung weight to body weight ratios was not discerned at 3 weeks but was evidenced at 4 weeks in the HomCKO mice; cardiomyocyte necrosis as well as the impairment of UPS performance as reflected by accumulation of a surrogate UPS substrate were not discernible until 3 weeks of age (Su et al., 2011a,b). Hence, to decipher potential molecular basis at the transcription level for CSN8 deficiency to induce cardiomyocyte necrosis and myocardial UPS impairment we elected to examine a time point before ( 2 weeks) and a time point after ( 3 weeks) these major pathologies become detectable in the Hom-CKO mice.

\section{Pathway and Network Analysis}

Differentially expressed genes (DEGs) were analyzed using the QIAGEN's Ingenuity Pathway Analysis (IPA, QIAGEN Redwood City, www.qiagen.com/ingenuity) to identify significantly enriched pathways. Expression data were processed by the IPA software suite system, which scores and ranks pathways enriched in our data by the proportion of pathway associated genes with significant expression values. Ultimately, this allows the 
visualization of canonical pathways significantly enriched for our given data set. Network analysis was performed using R package WGCNA on the expression profiles of all hand-curated pathway genes. The package WGCNA uses a topological overlap measure to construct weighted correlation networks. We applied it in this study to detect gene correlation modules, which are defined as groups of highly correlated genes. The network obtained was exported to Cytoscape, a network visualization software platform.

\section{Real Time Reverse Transcriptase PCR (RT' ${ }^{2}$-PCR)}

$\mathrm{RT}^{2}$-PCR was used to confirm the gene expression of Cops 8 and additional genes in mouse heart tissue. Predesigned primers and probes for $5^{\prime}$ UTR and exon 5 region of the CSN8 gene as well as for Synapsin II were obtained from Assays on Demand (Applied Biosystems). TaqMan Gold RT-PCR reagents were used to perform RT-PCR (Applied Biosystems, Foster City, CA). While the first-strand cDNA synthesis was carried out using a MultiScribe Reverse Transcriptase, AmpliTaq Gold DNA polymerase was used for DNA amplification. Triplicates of each RNA sample were performed. For control reactions, a no-reverse transcriptase (NRT) and a no-RNA control (NRC) for each experimental and reference gene (GAPDH) were included. Each well contained appropriate Master Mix pool or No-RT Master Mix pool $(19 \mu \mathrm{l})$ and appropriate RNA $(6 \mu \mathrm{l})$. Plate was sealed with a 96-well plate film and centrifuged at 1,200 $\times \mathrm{g}$ for $2 \mathrm{~min}$. Plate was then placed in StepOne Plus Real Time PCR Machine and program was run according to manufacturer's instruction. Changes in expression of each gene of interest were calculated relative to the endogenous control (housekeeping gene) GAPDH. An RNA concentration response curve was obtained for all genes including GAPDH. The validation curves were used to determine the concentration of RNA added to the RT-PCR reaction. Data from the $\mathrm{RT}^{2}$-PCR reactions were analyzed with use of $\mathrm{qBase}$ software (http://medgen.ugent.be/qbase/). This program uses a delta Ct (threshold cycle) relative quantitation model with PCR efficiency correction and multiple reference gene normalization.

\section{Western Blot Analysis}

Ventricular myocardial tissues from Hom-CKO mice as well as littermate controls were collected and immediately frozen in liquid nitrogen. Samples were then stored at $-80^{\circ} \mathrm{C}$ until further use. Tissues were homogenized using a lysis buffer $(80 \mathrm{mM}$ Tris$\mathrm{HCl}, 2 \% \mathrm{SDS}$ and $10 \%$ glycerol) and further sonicated on ice. The tissue lysates were boiled for $5 \mathrm{~min}$ and then centrifuged at $12,000 \mathrm{~g}$ for $8 \mathrm{~min}$. The supernatant was collected for western blot analyses. The concentration of total protein was determined with BCA reagents (Pierce, Rockford, IL, USA). To probe for FBXO31, $60 \mu \mathrm{g}$ of total proteins, and to probe for Asb-14 and TTLL1, 100 $\mu \mathrm{g}$ of total proteins were separated by SDS-PAGE, transferred to PVDF membrane, and blotted with rabbit anti-FBXO31 antibody (1:500, Abcam), Anti-Asb14 antibody (1:200, Santa Cruz), and anti-TTLL1 antibody (Santa Cruz, 1:1,000). Western blot analysis was performed as described previously (Su et al., 2011a).

\section{Statistical Analysis}

The microarray data were imported into an in-house analysis pipeline using Bioconductor for quantile normalization and statistical analysis. DEGs between groups at each time point or between time points within the group were revealed by $t$ test. Genes with $p<0.05$ after Benjamini and Hochberg false discovery rate correction for multiple hypothesis testing were considered as significant DEGs. The enrichment analysis of hand-curated pathway genes in significant DEGs was performed using Fisher's exact test. The $p$-value ( $<$ a cutoff value 0.05 ) obtained represents enrichment of pathway genes in the DEG set. The comparison of $\mathrm{RT}^{2}$-PCR and microarray data of individual gene expression among CTL, Het-CKO, Hom-CKO groups used one way ANOVA followed by Tukey's test for pair-wise comparison. Western blot data were analyzed with $t$-test using GraphPad Prism (GraphPad Software, San Diego, CA).

\section{RESULTS}

\section{Identification of DEGs in Response to CSN8-CKO}

Whole mouse genome DNA microarrays (CodeLink) were utilized to compare the gene expression profiles among control $\left(\mathrm{CSN} 8^{\text {flox/flox }}, \mathrm{CTL}\right)$, heterozygous CSN8-CKO (Het-CKO), and homozygous CSN8-CKO (Hom-CKO) mouse groups. The raw data for these DNA microarrays have been deposited in the National Center for Biotechnology Information Gene Expression Omnibus (NCBI GEO; www.ncbi.nlm.nih.gov/geo, Accession Number GSE100104) in accordance with Minimum Information About a Microarray Experiment (MIAME) standards (Brazma et al., 2001).

GeneSpring GS software (v.12.5) was initially used to analyze DEGs. The microarray data revealed 521 DEGs at 3-weeks-ofage with cut-off $\log 2 \mid$ fold change $\mid \geqq 1$. Among these genes, 271 genes were upregulated and 250 were downregulated. Similarly, at 2-weeks-of-age, out of 78 DEG, 35 genes were upregulated and 43 were downregulated.

The Hom-CKO mice start displaying cardiac hypertrophy and cardiac malfunction at 2 weeks of age and overt heart failure at 4 weeks ( $\mathrm{Su}$ et al., 2011b). One of the characteristics of cardiac hypertrophy or injury is reactivation of the fetal gene program. Using multiple methods, our group had previously shown the induction of fetal genes natriuretic peptide type A (Nppa) and B (Nppb), skeletal $\alpha$-actin (Acta1), and $\beta$-myosin heavy chain (Myh7) in the Hom-CKO hearts (Su et al., 2011b). In the present study, gene expression profiling also detected marked reactivation of the fetal gene program (Supplementary Figure $2 \mathrm{~A}$ ), which is consistent with cardiac pathology in the HomCKO mice and, more importantly here, provides a verification of the sensitivity as well as the accuracy of our microarray data. Down-regulation of calcium handling genes (e.g., Pln, Atp2a2) and adult forms of sarcomeric genes (e.g., Actc1 and Tpm1) is another gene expression signature of cardiac hypertrophy and failure. Our microarray data also revealed significant downregulation of these genes in Hom-CKO mouse hearts at 3 weeks (Supplementary Figure 2B), which is again consistent with 
known phenotype of Hom-CKO mice and once again attests the sensitivity and the accuracy of our microarray data.

Prolonged Cre transgene expression driven by the Myh6 promoter could have a significant impact on cardiac gene expression (Pugach et al., 2015), Het-CKO mice are therefore a better control group for the Hom-CKO mice than the CTL group in terms of controlling for the potential off-target effects of the transgenic Cre, given that both Het-CKO and Hom-CKO harbor Myh6-Cre and importantly that myocardial CSN8 protein levels are not discernibly altered in the Het-CKO mice compared with the littermate CTL (Supplementary Figure 1C) and wild type mice. Thus, the DEGs from the comparison between Het$\mathrm{CKO}$ and Hom-CKO reflect conservatively the impact of CSN8 deficiency on gene expression, thereby constituting the main stream data for our elucidation of their functional implication. From the Hom-CKO vs. Het-CKO comparison, the top underor over-expressed DEGs with respect to their fold change values along with $p$-values are listed in Tables 1, 2, respectively. The maximum down-regulation is by 77 and $91 \%$ at 2 and 3 weeks, respectively (Table 1), whereas the maximum up-regulation is 2.44 and 7.57 fold at 2 and 3 weeks, respectively (Table 2). For the most part, alterations of these DEGs are more pronounced at 3-weeks compared with 2-weeks, which is further unveiled by comparisons between the 3-week and the 2-week time points within each of the 3 genotypes. With rare exception, the expression levels of these top down- or up-regulated genes were comparable between 3- and 2-weeks-of-age within either CTL or Het-CKO genotypes (Tables 1, 2); however, the transcript levels of approximately $30 \%$ of the top down-regulated genes (Table 1) and $70 \%$ of the top up-regulated genes (Table 2) in the HomCKO mice differed significantly between the 3-week and 2-week time points.

We also used intersection analysis (Venn diagram) to identify shared DEGs among the three comparisons: Het-CKO vs. HomCKO, CTL vs. Het-CKO, and CTL vs. Hom-CKO, at both 2 and 3 weeks of age (Figure 1). The analyses reveal that at 2-weeksof-age (Figure 1A), 263, 241, and 319 genes had differential transcript expression for the three comparison pairs, respectively. These results indicate that 131 out of 263 DEGs were unique for Hom- vs. Het-CKO, 187 out of 241 were unique for Het-CKO vs. CTL, and 153 out of 319 were unique for Hom-CKO vs. CTL. There were 10 DEGs common between CTL vs. Het-CKO and Het- vs. Hom-CKO, 122 common between Het- vs. Hom-CKO and CTL vs. Hom-CKO, and 44 common between CTL vs. Hom$\mathrm{CKO}$ and CTL vs. Het-CKO. We did not find any DEGs that were common among all three comparisons at 2 weeks.

The 3-weeks-of-age Venn-diagram shows 286, 839, and 1003 DEGs for the Het- vs. Hom-CKO, CTL vs. Het-CKO, and CTL vs. Hom-CKO comparisons, respectively (Figure 1B). These results indicate that 177 out of 286 were unique for Het- vs. HomCKO, 295 out of 839 DEGs were unique for CTL vs. Het$\mathrm{CKO}$, and 415 out of 1003 were unique for CTL vs. HomCKO. There were 30 DEGs common between CTL vs. Het-CKO and Het vs. Hom-CKO, 509 common between CTL vs. HetCKO and CTL vs. Hom-CKO, and 74 common between CTL vs. Hom-CKO and Het vs. Hom-CKO. There were 5 DEGs that were common among all three comparisons at 3 weeks.
Those genes included cacna1s, ptgfr, mylk2 and ppp1r3g whereas one of them was an unknown gene (Supplementary Table 1 and Supplementary Figure 3). Of the four known genes, the expression differentials of the ptgfr and mylk2 genes between Hom-CKO and Het-CKO are more striking than the other two, and both are involved in cardiac muscle contraction. The ptgfr gene encodes the receptor for prostaglandin $\mathrm{F}_{2} \alpha\left(\mathrm{PGF}_{2} \alpha\right)$. $\mathrm{PGF}_{2} \alpha$ is produced during myocardial inflammation as a cardiac response (Tracey, 2002). In isolated cardiomyocytes, $\mathrm{PGF}_{2} \alpha$ has been shown to increase intracellular $\mathrm{Ca}^{2+}$ and therefore ventricular contractility in response to inflammation (Ponicke et al., 2000; Takayama et al., 2005) by increasing inositol triphosphate $\left(\mathrm{IP}_{3}\right)$ signaling pathway (Fabiato, 1992). Hence, the drastic down-regulation of ptgfr gene in Hom-CKO compared with both Het-CKO and CTL groups (Supplementary Figure 3B) may have contributed to CSN8-CKO-induced decrease in cardiac contractility. The mylk2 gene encodes for myosin light chain kinase (MLCK) enzyme that phosphorylates myosin light chain (MLC2). The phosphorylation of MLC2 regulates contraction of smooth muscles (Murthy, 2006) and increases the myosin-actin cross-bridge formation in cardiomyocytes following increased intracellular $\mathrm{Ca}^{2+}$ concentration and thereby improves the cardiac contractility (Moss and Fitzsimons, 2006; Stelzer et al., 2006). Of interest, the expression of mylk2 was modestly down-regulated in Het-CKO hearts but increased drastically in the Hom-CKO hearts (Supplementary Figure 3C), suggesting either that the down-regulation results from an offtarget effect of Myh6-Cre or that the increased mylk2 expression is a secondary effect. We have previously shown that CSN8deficiency in cardiomyocytes leads to cardiac hypertrophy and heart failure (Su et al., 2011b, 2013), but the direct effect of CSN8 function on ventricular contractility was not assessed at the transcriptional level. Hence, this is the first time it is proposed that decreased transcription of ptgfr contributes to CSN8-CKOinduced decrease in cardiac contractility.

The findings that very few DEGs were common to all three pairwise comparisons ( 0 at 2 weeks and up to 5 at 3 weeks) also indicate that virtually none of the DEGs caused by loss of CSN8 proteins (Hom-CKO vs. Het-CKO) overlap with those caused potentially by the off-target effects of Cre (Het-CKO vs. CTL).

In addition, we performed longitudinal comparisons between the 2- and 3-week time points within each of the three genotypes, which identified 6, 74, and 1626 significant DEGs for the CTL, Het-CKO, and Hom-CKO mice, respectively. Among these DEGs, only 1 DEG is common to all 3 genotypes, 24 additional DEGs are shared between Het-CKO and Hom-CKO, 1 additional DEGs is common between CTL and Hom-CKO but no additional DEG common between CTL and Het-CKO (Figure 1C). From 2- to 3-week, very few significant DEGs were found in either CTL or Het-CKO mice while a large number of significant DEGs detected in Hom-CKO mice with only a very small proportion of them shared among different genotypes, indicating that the effect of perinatal CSN8 deficiency has a great impact on myocardial transcriptome independent of developmental changes during this critical period; this also suggests that the selection of this time window is physiologically appropriate for revealing the genomic effect of perinatal CSN8 deficiency. 
TABLE 1 | List of top down-regulated DEGs in Hom-CKO vs. Het-CKO and their temporal changes within each genotype.

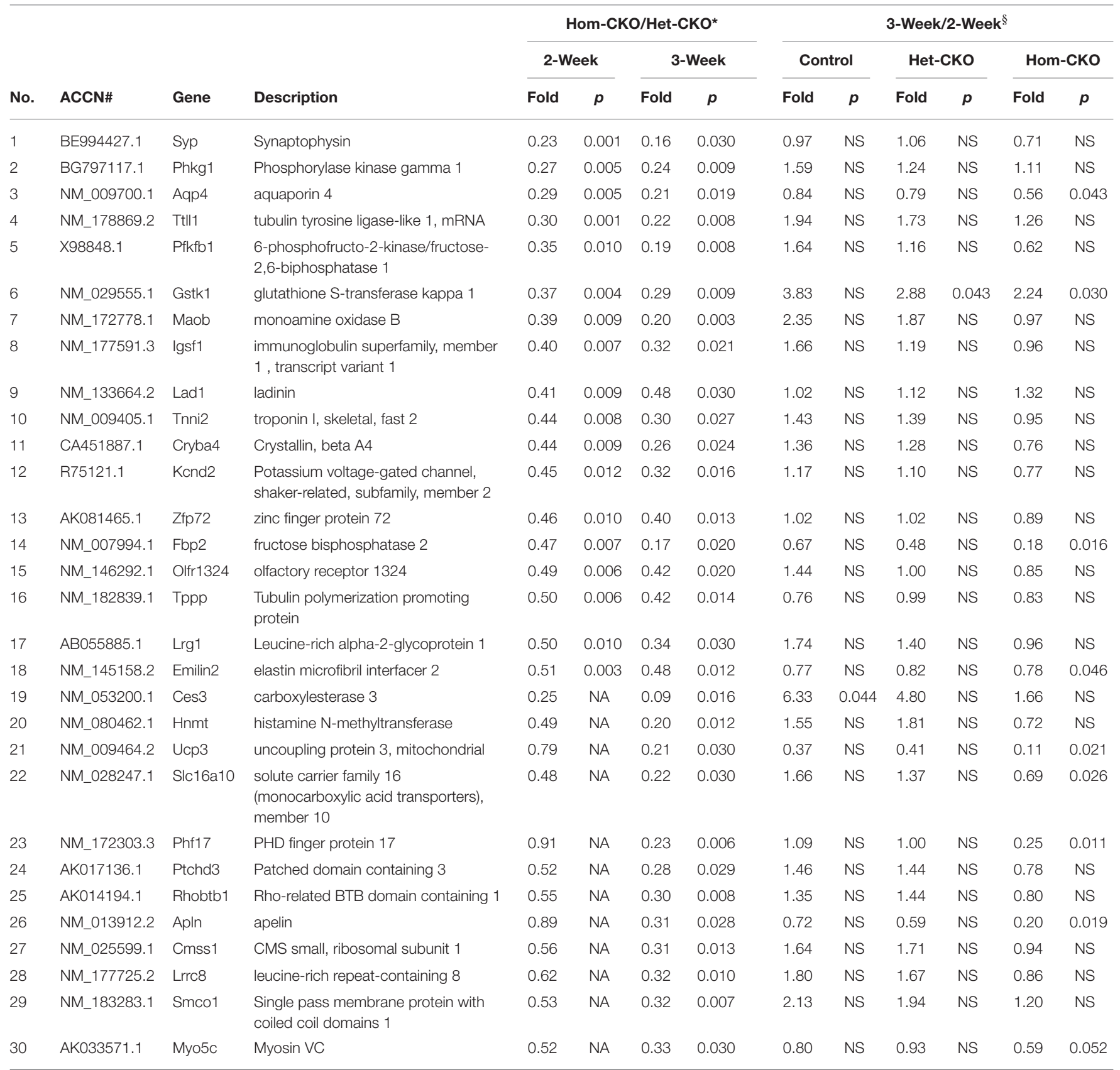

*In the Hom-CKO vs. Het-CKO comparisons, p-vales were derived from one way ANOVA followed by Tukey's test where other two pair-wise comparisons (Hom-CKO vs. CTL and Het-CKO vs. CTL) were not shown; NA, not available as statistical significance was not shown by ANOVA. ${ }^{\S}$ For comparisons between the 3-and. 2-week time points within each genotype, $p$-values were derived from t-test; NS, not significant. The footnote remains the same for Table 2.

\section{Functional Enrichment Analysis for Hand-Curated Pathway Genes}

To determine the functional enrichment of certain pathways, we performed Fisher's exact test of hand-curated pathway genes. A pathway with a $p$-value below 0.05 was considered as a functionally enriched pathway. Based on our previous findings of CSN8 function, we analyzed CRL SRs (SOCS and Fbox genes), autophagy, microtubule-related, vesicle trafficking, chromatin remodeling, endocytosis, ATP synthases, and cell death pathways. The results from the Hom-CKO vs. HetCKO comparison showed that except the cell death pathway, all pathway genes were functionally enriched in response to CSN8 deficiency in mouse hearts at both 2 and 3 weeks (Table 3). The hand-curated individual genes for all of the above nine pathways are listed in Supplementary Tables 2-10. 
TABLE 2 | List of top up-regulated DEGs in Hom-CKO vs. Het-CKO and their temporal changes within each genotype.

\begin{tabular}{|c|c|c|c|c|c|c|c|c|c|c|c|c|c|}
\hline \multirow[b]{3}{*}{ No. } & \multirow[b]{3}{*}{ ACCN\# } & \multirow[b]{3}{*}{ Gene } & \multirow[b]{3}{*}{ Description } & \multicolumn{4}{|c|}{ Hom-CKO/Het-CKO* } & \multicolumn{6}{|c|}{ 3-Week/2-Week ${ }^{\S}$} \\
\hline & & & & \multicolumn{2}{|c|}{ 2-Week } & \multicolumn{2}{|c|}{ 3-Week } & \multicolumn{2}{|c|}{ Control } & \multicolumn{2}{|c|}{ Het-CKO } & \multicolumn{2}{|c|}{ Hom-CKO } \\
\hline & & & & Folds & $p$ & Folds & $p$ & Folds & $p$ & Folds & $p$ & Folds & $p$ \\
\hline 1 & NM_153744.1 & Prkag3 & $\begin{array}{l}\text { protein kinase, AMP-activated, } \\
\text { gamma } 3 \text { non-catatlytic subunit }\end{array}$ & 2.44 & 0.006 & 4.16 & 0.010 & 1.20 & NS & 1.01 & NS & 1.72 & NS \\
\hline 2 & NM_173385.1 & Cilp & $\begin{array}{l}\text { cartilage intermediate layer protein, } \\
\text { nucleotide pyrophosphohydrolase }\end{array}$ & 2.44 & 0.013 & 4.18 & 0.020 & 0.63 & NS & 0.82 & NS & 1.41 & NS \\
\hline 3 & BQ176278.1 & Grm1 & Glutamate Receptor,metabotropic 1 & 2.44 & 0.009 & 2.38 & 0.030 & 1.66 & NS & 1.74 & NS & 1.70 & NS \\
\hline 4 & NM_183294.1 & Cdkl1 & $\begin{array}{l}\text { cyclin-dependent kinase-like } 1 \\
\text { (CDC2-related kinase) }\end{array}$ & 2.43 & 0.007 & 3.98 & 0.005 & 1.00 & NS & 0.88 & NS & 1.44 & 0.031 \\
\hline 5 & BC052064.1 & Parp6 & $\begin{array}{l}\text { Poly (ADP-ribose)polymerase family, } \\
\text { member } 6\end{array}$ & 2.39 & 0.002 & 3.72 & 0.007 & 1.09 & NS & 1.13 & NS & 1.76 & 0.026 \\
\hline 6 & $\mathrm{~B} \mid 412344.1$ & Mettl21d & Methyltransferase like 21D & 2.37 & 0.005 & 3.05 & 0.030 & 1.02 & NS & 1.34 & NS & 1.72 & 0.031 \\
\hline 7 & NM_021467.4 & Tnni1 & troponin I, skeletal, slow 1 & 2.31 & 0.022 & 1.53 & NA & 0.11 & NS & 0.36 & NS & 0.24 & 0.043 \\
\hline 8 & NM_007669.2 & Cdkn1a & $\begin{array}{l}\text { cyclin-dependent kinase inhibitor } 1 \mathrm{~A} \\
\text { (P21) }\end{array}$ & 2.27 & 0.007 & 6.34 & 0.005 & 0.87 & NS & 1.25 & NS & 3.50 & 0.025 \\
\hline 9 & NM_019875.1 & Abcb9 & $\begin{array}{l}\text { ATP-binding cassette, sub-family B } \\
\text { (MDR/TAP), member } 9\end{array}$ & 2.25 & 0.004 & 3.61 & 0.030 & 1.11 & NS & 0.93 & NS & 1.49 & 0.046 \\
\hline 10 & NM_016867.1 & Semcap2 & $\begin{array}{l}\text { semaF cytoplasmic domain } \\
\text { associated protein } 2\end{array}$ & 2.22 & 0.007 & 4.87 & 0.007 & 1.21 & NS & 1.42 & 0.044 & 3.11 & 0.027 \\
\hline 11 & NM_013468.2 & Ankrd1 & $\begin{array}{l}\text { ankyrin repeat domain } 1 \text { (cardiac } \\
\text { muscle) }\end{array}$ & 3.53 & 0.119 & 7.57 & 0.011 & 1.45 & NS & 1.06 & NS & 2.28 & NS \\
\hline 12 & NM_013681.1 & Syn2 & synapsin II & 4.22 & 0.520 & 7.38 & 0.010 & 1.50 & NS & 1.12 & NS & 1.96 & NS \\
\hline 13 & NM_019687.2 & Slc22a4 & $\begin{array}{l}\text { solute carrier family } 22 \text { (organic cation } \\
\text { transporter), member } 4\end{array}$ & 1.42 & NA & 6.48 & 0.013 & 3.37 & NS & 0.83 & NS & 3.77 & 0.016 \\
\hline 14 & CB848957.1 & Auh & $\begin{array}{l}\text { AU RNA binding protein/enoyl CoA } \\
\text { hydratase }\end{array}$ & 1.78 & NA & 6.25 & 0.004 & 1.03 & NS & 0.94 & NS & 3.31 & 0.014 \\
\hline 15 & Al853801.1 & Srsf1 & Serine-arginine-rich splicing factor 1 & 2.78 & NA & 6.21 & 0.002 & 1.25 & NS & 1.44 & NS & 3.23 & 0.015 \\
\hline 16 & NM_010077.1 & Drd2 & dopamine receptor 2 & 1.61 & NA & 6.05 & 0.012 & 1.22 & NS & 1.07 & NS & 4.01 & 0.041 \\
\hline 17 & AA536960.1 & Rexo1 & RNA exonuclease 1 homolog & 1.10 & NA & 5.79 & 0.008 & 1.22 & NS & 1.08 & NS & 5.68 & 0.028 \\
\hline 18 & BC027359.1 & Mphosph8 & M phase phosphoprotein 8 & 2.72 & 0.002 & 5.45 & 0.008 & 0.76 & NS & 0.77 & NS & 1.54 & NS \\
\hline 19 & NM_011103.1 & Prkcd & protein kinase $\mathrm{C}$, delta & 2.92 & 0.001 & 5.40 & 0.004 & 1.19 & NS & 1.11 & NS & 2.06 & 0.017 \\
\hline 20 & W83574.1 & Ndufa3 & $\begin{array}{l}\text { NADH dehydrogenase (ubiquinone) } 1 \\
\text { alpha subcomplex, } 3\end{array}$ & 2.59 & 0.002 & 5.23 & 0.014 & 0.89 & NS & 0.94 & NS & 1.89 & 0.014 \\
\hline 21 & NM_011485.3 & Star & steroidogenic acute regulatory protein & 1.68 & NA & 5.15 & 0.030 & 1.16 & NS & 0.83 & NS & 2.56 & NS \\
\hline 22 & NM_145495.1 & Rin1 & Ras and Rab interactor 1 & 2.03 & NA & 4.96 & 0.005 & 1.46 & NS & 1.07 & NS & 2.60 & 0.026 \\
\hline 23 & NM_007474.1 & Aqp8 & aquaporin 8 & 2.07 & NA & 4.81 & 0.030 & 1.12 & NS & 1.14 & NS & 2.66 & NS \\
\hline 24 & NM_199222.1 & Lman1I & Lectin, mannose-binding 1 like & 1.42 & NA & 4.77 & 0.013 & 1.24 & NS & 1.16 & NS & 3.88 & 0.013 \\
\hline 25 & Al647986.1 & Bhmt2 & $\begin{array}{l}\text { Betaine-homocysteine } \\
\text { methyltransferase } 2\end{array}$ & 2.72 & 0.004 & 4.60 & 0.006 & 0.91 & NS & 1.15 & NS & 1.94 & 0.044 \\
\hline 26 & BY457927.1 & Rps3a1 & Ribosomal protein S3a & 1.21 & NA & 4.52 & 0.008 & 1.04 & NS & 1.12 & NS & 4.17 & 0.016 \\
\hline 27 & AK009352.1 & Nmrk2 & Nicotinamide riboside kinase 2 & 2.46 & 0.017 & 4.39 & 0.028 & 1.08 & NS & 1.25 & NS & 2.24 & NS \\
\hline 28 & NM_080640.3 & Baalc & $\begin{array}{l}\text { brain and acute leukemia, } \\
\text { cytoplasmic }\end{array}$ & 1.22 & NA & 4.38 & 0.030 & 1.15 & NS & 1.08 & NS & 3.87 & 0.037 \\
\hline 29 & NM_024286.1 & Popdc3 & popeye domain containing 3 & 1.91 & NA & 4.28 & 0.005 & 1.41 & NS & 1.00 & NS & 2.23 & 0.025 \\
\hline 30 & BE911434.1 & Fam20a & $\begin{array}{l}\text { Family with sequence similarity } 20 \text {, } \\
\text { member A }\end{array}$ & 1.41 & NA & 4.23 & 0.003 & 1.00 & NS & 0.98 & NS & 2.93 & 0.019 \\
\hline
\end{tabular}

\section{Hierarchical Cluster Analysis of DEGs between CTL and CSN8-CKO Samples}

A heat map provides an immediate visual summary of experimental data since the individual values, represented by colors, indicate low and high levels of transcript abundances (Kalil and Florescu, 2013). In hierarchical clustering, subjects with similar features in a heatmap can be grouped as clusters (Zhang et al., 2017). For example, in terms of gene array, genes with similar expression can be clustered in the same group while similarity among clusters is shown by dendrograms. We used average linkage and Euclidean distance metric to generate hierarchical clustering. The results of the clustering 


\section{A}

Het- vs. Hom-CKO (263) CTL vs. Het-CKO (241)

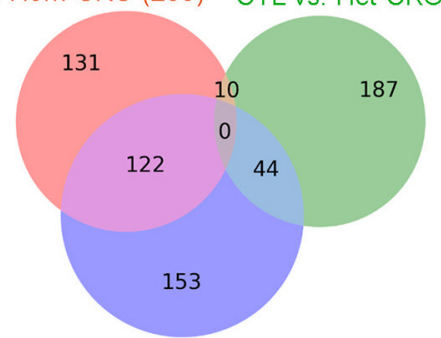

CTL vs. Hom-CKO (319)
B

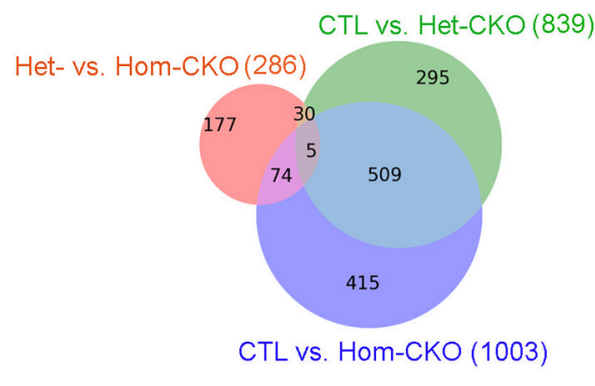

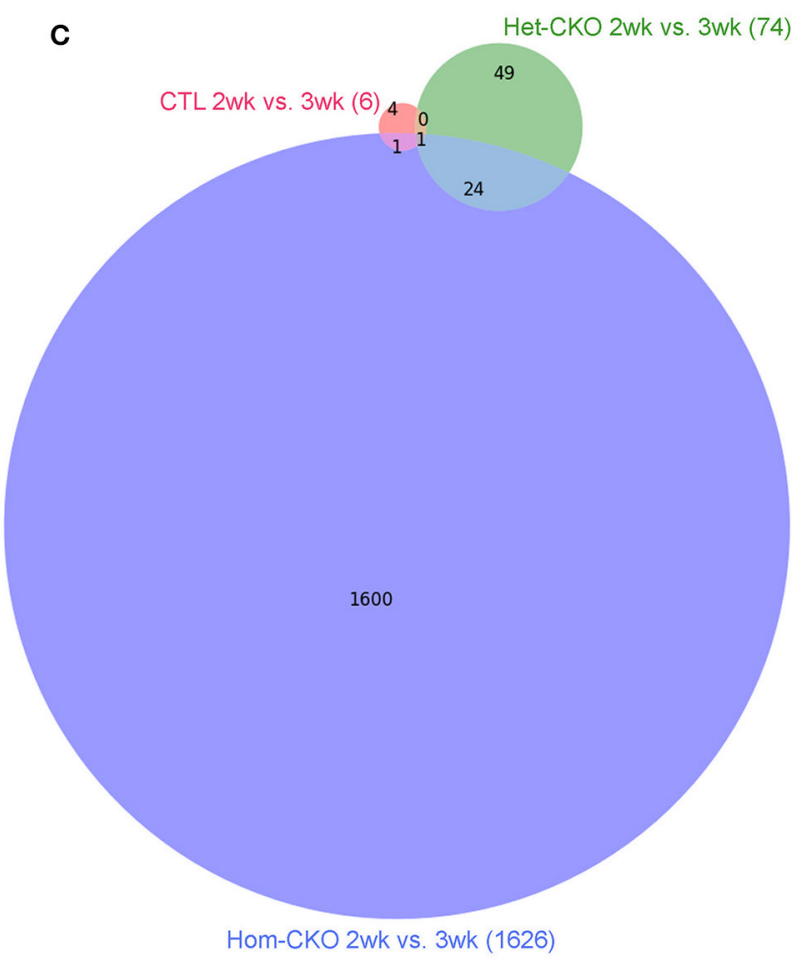

FIGURE 1 | Venn diagrams showing the distribution of significant differentially-expressed genes (DEGs) that are unique or common among the three comparisons between genotypes: Het-CKO vs. Hom-CKO (Red), Control (CTL) vs. Hom-CKO (blue), CTL vs. Het-CKO (green) at postnatal 2 weeks (A) and 3 weeks (B), or among the three comparisons between the 2-weeek (2wk) and 3-week (3wk) time points within each genotype (C).

analysis of all DEGs among CTL, Het- and Hom-CKO groups at 2- and 3-weeks-of-age are displayed in Supplementary Figure 4. The functional enrichment analysis for hand-curated genes determined DEGs within autophagy, CRL SRs (F-box and SOCSbox genes), cell death pathway, chromatin remodeling, vesicle trafficking, and microtubule-related pathway genes. Using those DEGs, we created a heat map to look at hierarchical clustering among pathways that are all associated to CSN8 function (Figure 2). The gene expression profile of Hom-CKO group was significantly different from CTL and Het-CKO group and DEGs from different pathways could be successfully clustered. Compared with CTL and Het-CKO group, we identified 18 hand-curated genes that were downregulated and 19 genes that were upregulated in Hom-CKO group. Heatmap/hierarchical clustering also showed that out of 37 DEGs, 18 were microtubulerelated genes, 8 were cell-death pathway genes, three were Fbox genes, two were SOCS-box genes, two genes for fetal gene program and chromatin remodeling, and a single gene for vesicular trafficking and autophagy pathways (Figure 2).

\section{Ingenuity Pathway Analysis}

QIAGEN's IPA was employed to identify integral biological pathways, cellular processes and upstream regulators that are associated with the cardiac CSN8 deficiency. For reasons described earlier, the significant DEGs from the Het-CKO vs. Hom-CKO comparison best reflect the impact of CSN8 


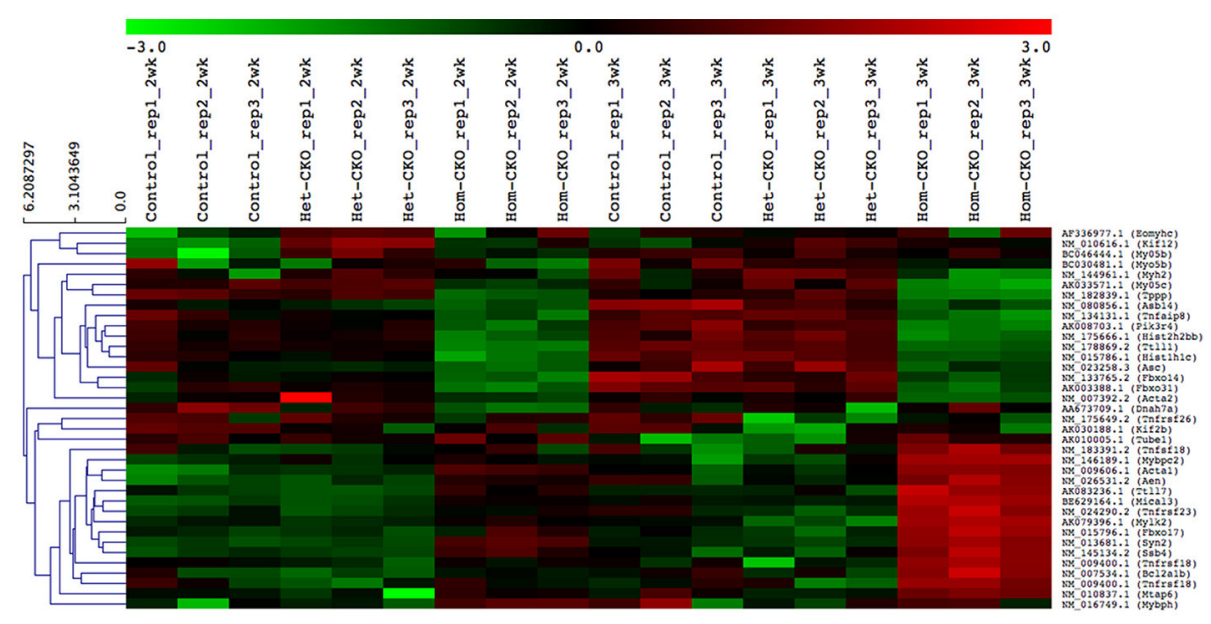

FIGURE 2 | Heatmap/hierarchical clustering of DEGs of seven different pathways. All replicates of each group (Control, Het-CKO and Hom-CKO) are shown. Similarity between clusters is shown through dendrograms. Vertical dendrograms represent expression values from microarray data. Green and red indicate low and high levels of transcript abundance, respectively.

deficiency on gene expression. Thus, we will describe IPA results from this cohort of comparisons in greater detail here. IPA shows that at 2 weeks of age, DEGs in Hom-CKO compared with littermate Het-CKO are significantly enriched in a large number of pathways, some of which are predicted to have increased activity (i.e., positive $\mathrm{z}$-score), including ERK/MAPK signaling, synaptic long term potentiation, GNRH signaling, P2Y purinergic receptor signaling pathway, dopamineDARPP32 feedback in cAMP signaling, NF- $\kappa$ B signaling, CREB signaling, production of $\mathrm{NO}$ and ROS in macrophages, and IL-8 signaling (Figure 3A). Activation of these pathways likely plays a major role in mediating cardiac hypertrophy observed at this time point and/or the inflammatory responses and cell death that become apparent at a later time point (e.g., 3 weeks). Enhanced ERK/MAPK signaling is well-known to perturb cardiac development (Bezniakow et al., 2014) and to participate in both adaptive and maladaptive cardiac hypertrophy and remodeling (Rose et al., 2010). CREB is a transcription factor implicated in cardiac hypertrophy that can be activated by a variety of upstream signaling pathways including ERK/MAPK (Subedi et al., 2017). The involvement of purinergic signaling, including $\mathrm{P} 2 \mathrm{Y}$ receptor-mediated signaling in cardiovascular pathophysiology, such as cardiac hypertrophy and heart failure, and its emergence as a therapeutic target are well-evidenced in recent literature (Burnstock, 2017). A meta-analysis of genomewide association studies has revealed that dopamine-DARPP32 feedback in cAMP signaling is one of the signaling pathways shared by mood disorders and cardio-metabolic diseases (Amare et al., 2017). Meanwhile, NF- $\kappa \mathrm{B}$ signaling, production of $\mathrm{NO}$ and ROS in macrophages, and IL- 8 signaling are apparently involved in the regulation of inflammation and cell death (Valen, 2011). Both inflammation and cell death are clearly evidenced in Hom-CKO hearts at 3 weeks of age (Su et al., 2011a,b).

For the 3-week time point, IPA reveals that significant DEGs between Hom-CKO and Het-CKO are enriched in fewer pathways (Figure 3B) although the total number of DEGs is somewhat larger at 3-weeks (286) than at 2-weeks (263) (Figures 1A,B). Nevertheless, approximately one third of the enriched pathways at 3-weeks had shown enrichment at 2-weeks as well, with some of their activity changes being more predictable at 3-weeks. For example, NRF2-mediated oxidative stress response was statistically the most significant pathway at 2 -weeks and its enrichment remained significant at 3-weeks but the pathway activity was predicted from being unchanged ( $\mathrm{z}$-score $=0$ ) at 2 -weeks to being increased at 3-weeks (positive $\mathrm{z}$-score). Similarly, the aryl hydrocarbon receptor (AhR) signaling was predicted from being unchanged at 2-weeks to being decreased at 3 -weeks (negative $\mathrm{z}$-score). The AhR is a transcription factor of the basic helix-loophelix/PER-ARNT-SIM family. It can be activated by both environmental factors (e.g., polycyclic aromatic hydrocarbons or dioxins) and endogenous ligands (e.g., tryptophan derivatives, arachidonic acid metabolites, equilenin, heme metabolites, and indigoids). Ligand-bound or activated AhR moves to the nucleus where it forms heterodimers with aryl hydrocarbon receptor nuclear translocator (ARNT). The AhR/ARNT heterodimer binds to xenobiotic responsive elements (XREs) or dioxin responsive elements (DREs) in the enhancer sequences of genes encoding phase I and II xenobiotic metabolizing enzymes, such as cytochrome P450 monooxygenases (CYP1A1, CYP1A2, and CYP1B1) and glutathione-S-transferases (GSTs), NADPH/quinone oxidoreductase (NQO1), and aldehyde dehydrogenase 3, and activates these genes (Dietrich, 2016). Decreased AhR signaling in Hom-CKO hearts may conceivably diminish the ability to detoxify exogenous and endogenous toxic factors, which may contribute to cardiac dysfunction and cardiomyocyte death. Beyond activating the canonical xenobiotic metabolism signaling, AhR can also trigger the antioxidant response through transactivating the NRF2 gene and collaborating with NRF2 to activate NRF2 target genes 
TABLE 3 | The $p$-value of hand-curated pathway genes tested with Fisher's Exact test.

\begin{tabular}{|c|c|c|c|c|c|}
\hline \multirow[t]{2}{*}{ No. } & \multirow[t]{2}{*}{ Pathways } & \multicolumn{2}{|c|}{2 weeks } & \multicolumn{2}{|c|}{3 weeks } \\
\hline & & Hom-CKO vs. CTL & Hom-CKO vs. Het-CKO & Hom-CKO vs. CTL & Hom-CKO vs. Het-CKO \\
\hline 1 & SOCS-box & 0.002256 & $2.24 \mathrm{E}-06$ & 9.70E-07 & 1.36E-08 \\
\hline 2 & F-box & $4.78 \mathrm{E}-09$ & $1.53 \mathrm{E}-14$ & $1.82 \mathrm{E}-09$ & $1.20 \mathrm{E}-15$ \\
\hline 3 & Autophagy & $1.39 \mathrm{E}-11$ & $2.20 \mathrm{E}-16$ & $6.44 \mathrm{E}-16$ & $2.20 \mathrm{E}-16$ \\
\hline 4 & Microtubules-related & $2.20 \mathrm{E}-16$ & $2.20 \mathrm{E}-16$ & $2.20 \mathrm{E}-16$ & $2.20 \mathrm{E}-16$ \\
\hline 5 & Vesicle trafficking & $1.39 \mathrm{E}-11$ & $2.20 E-16$ & $2.20 \mathrm{E}-16$ & $2.20 E-16$ \\
\hline 6 & Chromatin Remodeling & 6.90E-09 & $2.20 \mathrm{E}-16$ & $2.20 \mathrm{E}-16$ & $2.09 \mathrm{E}-15$ \\
\hline 7 & Endocytosis & 8.46E-06 & $2.20 \mathrm{E}-16$ & 7.33E-15 & $1.84 \mathrm{E}-14$ \\
\hline 8 & ATP synthases & 0.1417 & 6.05E-06 & 4.04E-06 & 0.006758 \\
\hline 9 & Cell Death Pathway & 1 & 1 & 1 & 1 \\
\hline
\end{tabular}

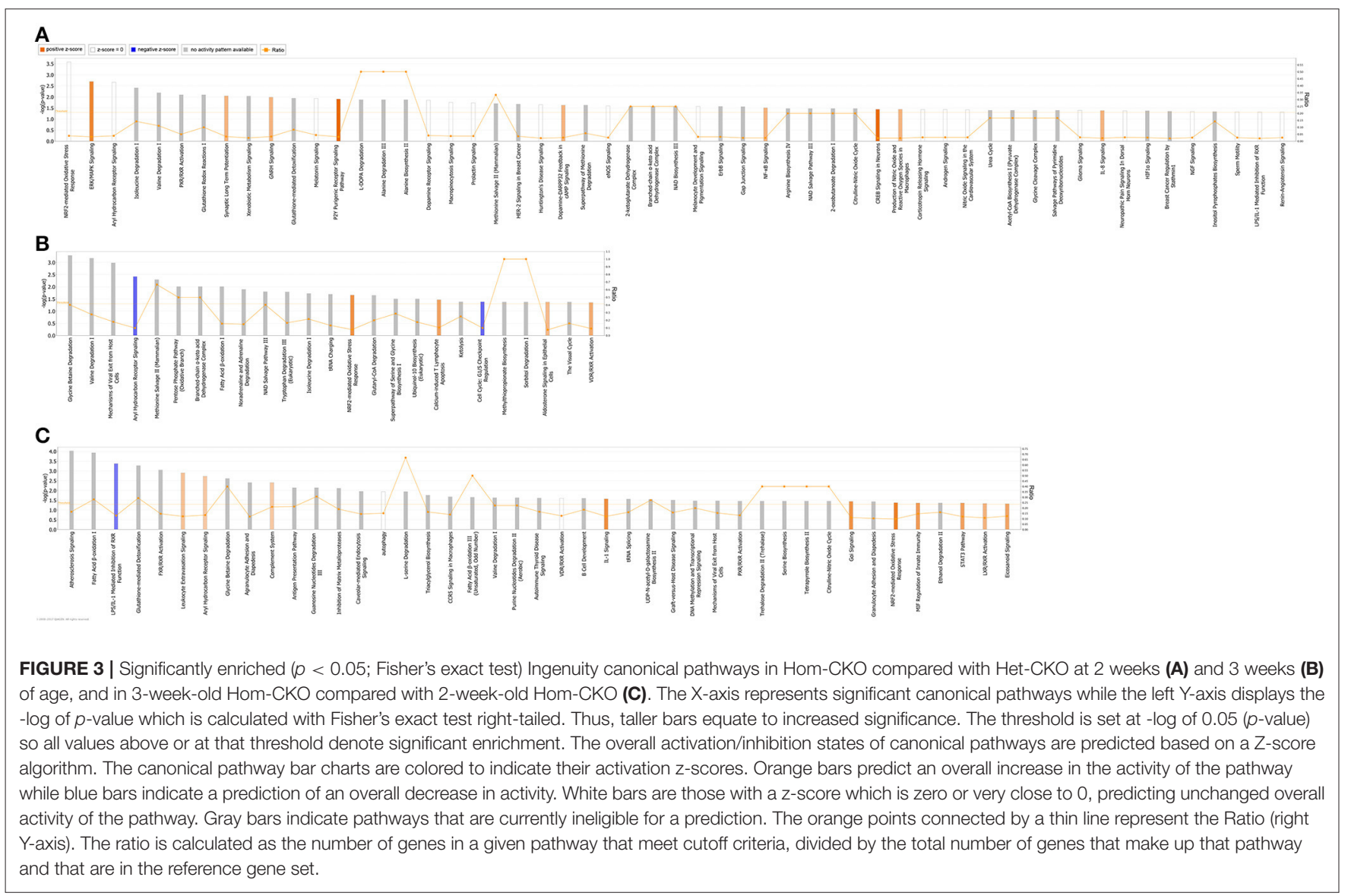

(Dietrich, 2016). The NRF2-mediated oxidative stress response is a well-known master pathway that enables the cell to deal with harmful stress including oxidative stress. The enrichment of DEGs in these pathways related to oxidative stress responses strongly suggests that cardiac CSN8 deficiency increases oxidative stress and something alike which in turn trigger cellular defense against these toxic stresses. This is supported by a prior transcription profiling study on Drosophila deficient of a CSN subunit which also indicates that CSN regulates the redox signaling pathways (Oron et al., 2007). This is further corroborated by our prior findings that increased myocardial protein carbonyls and massive cardiomyocyte necrosis are observed in mice with cardiac deficiency of CSN8 (Su et al., 2011a,b, 2013). Enrichment of DEGs in the sperm motility pathway is consistent with dysregulated microtubule functioning that is evidenced by perturbed vesicle trafficking (Table 3) and impaired autophagosome maturation in Hom-CKO mouse hearts (Su et al., 2011a). Comparisons between Hom-CKO and CTL and between Het-CKO and CTL are shown in Supplementary Figures 5, 6, respectively. 
We also performed IPA analysis on DEG data generated from comparison of gene expression between 3- and 2weeks of age within each genotype (i.e., data summarized in Figure 1C). The analysis reveals that there were 4 and 12 pathways with statistically significant enrichment of the DEGs in the CTL and Het-CKO mice, respectively but the overall activity of these pathways was either unchanged or not predictable (Supplementary Figure 7). By sharp contrast, this analysis has identified in the Hom-CKO group many more pathways with significant enrichment of DEGs, with 10 of them showing increased activity and one showing decreased activity (Figure 3C). Notably, 7 of the 10 activity-increased pathways (e.g., leukocyte extravasation signaling, complement system, IL-1 signaling, Gai signaling, MIF regulation of innate immunity, STAT3 pathway, and eicosanoid signaling) and the only activity-decreased pathway (i.e., LPS/IL-1 mediated inhibition of RXR function) are known to fall in the upstream signaling, the execution, or the downstream signaling of necrosis. The remaining 3 activity-increased pathways (e.g., AhR signaling, NRF2-mediated oxidative stress response, and LXR/RXR activation) are known to deal with xenobiotic metabolism and oxidative stress. Hence, these longitudinal changes in pathway genes correlate extremely well to the observation that massive cardiomyocyte necrosis occurs in Hom-CKO hearts but it was not discernible until 3 weeks of age (Su et al., 2011a,b).

\section{Network Analysis of Hand-Curated Pathway Genes}

The co-expression network of hand-curated pathway genes is shown in Supplementary Figure 8. To generate this network, we used array data for hand-curated pathway genes. The coexpression network helped us to identify interactions among genes of same or different pathways. Hence, each module/cluster in the co-expression network, designated with different colors, represents genes from multiple pathways that showed similar expression patterns (Supplementary Figure 8). There were 13 different modules identified in our co-expression network with blue module and turquoise module as the two densest modules containing the majority of differentially expressed pathway genes (Table 4). The differentially expressed pathway genes usually had more interacting neighbors, indicating their pivotal roles in influencing other genes. In total, network analyses identified co-expression of 28 DEGs (Triangle) along with some genes that are missing in statistical analysis (Circle). For example, Pam, Sqstm1, Tpm2 in the blue module, and Traf3, Myo9b, C1qtnf4, Bcl2, Bcl10, Myl9, Dnalc4, Kif5b, Tnfsf5ip1, Eml1 in the turquoise module could be genes affected by CSN8-CKO. These genes, although not detected as significant DEGs from our gene array data, are in the same module of most of the differentially expressed pathway genes, and have larger numbers of interacting neighbors, including those differentially expressed pathway genes (Supplementary Figure 8). Table 4 shows the total number of genes in each identified module from network analysis, and the corresponding number of genes of each pathway enriched in each module. For example, in the blue module; the genes with the most interacting partners includes Syn2 (trafficking), ssb4 (SOCS-box), Mical3 and Tppp (microtubules) with 53, 49, 47, and 46 interactions, respectively. Similarly Tnfaip8 (cell death), Ttll1 (microtubules), Hist1h1c and Hist $2 h 2 b b$ (chromatin remodeling) showed 60, 52, and 51 interactions in the turquoise module, respectively. These genes do not necessarily interact with DEGs only but also with non-DEGs genes (Supplementary Figure 8). In summary, the co-expression network map demonstrates that CSN8-CKO-induced effects on CRLs substrates of UPS, autophagy, cell death, vesicle trafficking, chromatin remodeling and microtubule-related pathways are all interlinked. CSN8-induced transcriptional effects on genes of one pathway can have direct or indirect effects on other pathways.

\section{DEGs Induced by CSN8 Deficiency Enrich in the Chromatin Remodeling Pathway}

The eukaryotic DNA is packaged around histone proteins in a structure called chromatin. In order for transcriptional regulators to get access to genomic DNA, chromatin structure needs to be modulated via a process known as chromatin remodeling (Hughes and Rando, 2014). As shown in Table 3, chromatin remodeling is one of the processes with significant enrichment of DEGs in Hom-CKO hearts. Histone proteins act as crucial regulators of transcription through chromatin remodeling. Hence, we paid particular attention to expression changes of histone gens. In CSN8$\mathrm{CKO}$ myocardium, not only was the expression of two of the histone genes Hist $1 h 1 c$ and Hist2h2bb, significantly affected (Figure 4), but these two DEGs were also shown to have the most number of interactions with other pathway genes (Supplementary Figure 8), indicative of a significant impact of CSN8 deficiency on transcriptional regulation.

\section{CSN8 Deficiency Decreased the Transcript Levels of CRL Substrate Receptors}

A major purported role for CSN-mediated cullin deneddylation to regulate CRLs is to prevent autoubiquitination and subsequent destruction of SR proteins of the CRL complexes, thereby preserving SRs of CRLs (Wee et al., 2005; Wang and Martin, 2015). Indeed, the decreased protein levels of SRs were observed in cells and tissues deficient of CSN subunits (Wee et al., 2005; Su et al., 2011b). We have previously observed in Csn8deficient heart and liver tissues that the deneddylase activity of the CSN is compromised, which results in significantly decreased protein levels of various SR molecules (Lei et al., 2011, 2013; Su et al., 2011b, 2013). Here, we analyzed the mRNA levels of several families of SR genes in response to CSN8CKO (Table 3, Figure 5). The expression of SR genes Fbxo31, Klhl41 and Klhdc1, was significantly lower in Hom-CKO heart as compared to CTL and Het-CKO counterparts at both 2and 3 -weeks-of-age (Figures 5A-C). Similarly, we found that at 2- and 3-weeks-of-age, the expression of Fbxo14 SR gene was significantly lower in Hom-CKO heart when compared to CTL but not to heterozygous $\mathrm{KO}$ heart (Figure 5D). The 
TABLE 4 | Distribution of various pathway genes in each module of co-expression network.

\begin{tabular}{|c|c|c|c|c|c|c|c|c|c|c|c|}
\hline No. & Module & Total genes & DEGs & Cell death & Microtubules & Fetal genes & Autophagy & Vesicle trafficking & $\begin{array}{l}\text { SOCS } \\
\text { box }\end{array}$ & F-box & $\begin{array}{l}\text { Chromatin } \\
\text { remodeling }\end{array}$ \\
\hline 1 & Turquoise & 50 & 10 & 15 & 18 & 1 & 4 & 4 & 2 & 1 & 5 \\
\hline 2 & Blue & 46 & 15 & 15 & 13 & 3 & 5 & 3 & 2 & 2 & 3 \\
\hline 3 & Brown & 23 & 0 & 3 & 15 & 0 & 1 & 2 & 1 & 0 & 1 \\
\hline 4 & Yellow & 20 & 1 & 10 & 9 & 0 & 0 & 0 & 0 & 1 & 0 \\
\hline 5 & Green & 12 & 0 & 3 & 6 & 0 & 2 & 1 & 0 & 0 & 0 \\
\hline 6 & Red & 11 & 0 & 5 & 4 & 0 & 1 & 1 & 0 & 0 & 0 \\
\hline 7 & Magenta & 9 & 1 & 2 & 5 & 0 & 0 & 1 & 0 & 0 & 1 \\
\hline 8 & Greenyellow & 8 & 0 & 3 & 5 & 0 & 0 & 0 & 0 & 0 & 0 \\
\hline 9 & Black & 8 & 0 & 4 & 3 & 0 & 0 & 1 & 0 & 0 & 0 \\
\hline 10 & Pink & 7 & 0 & 2 & 5 & 0 & 0 & 0 & 0 & 0 & 0 \\
\hline 11 & Gray & 6 & 0 & 2 & 3 & 0 & 1 & 0 & 0 & 0 & 0 \\
\hline 12 & Purple & 6 & 0 & 3 & 3 & 0 & 0 & 0 & 0 & 0 & 0 \\
\hline 13 & Tan & 5 & 1 & 2 & 1 & 0 & 1 & 0 & 0 & 1 & 0 \\
\hline
\end{tabular}

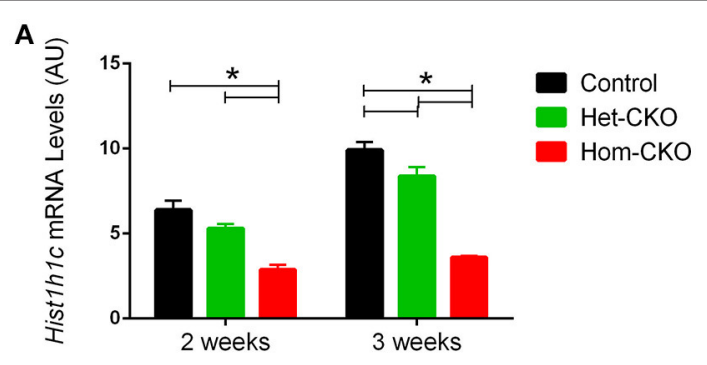

B

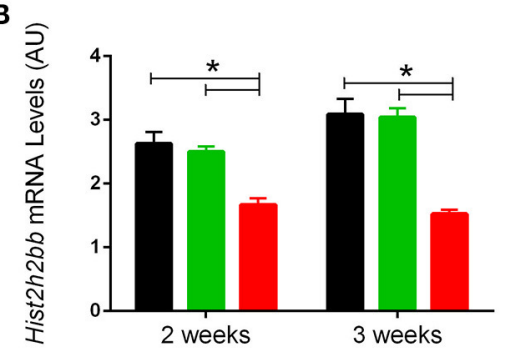

FIGURE 4 | Microarray-derived mRNA expression levels of the Hist1h1c (A) and Hist2h2bb genes (B) in CTL, Het-CKO, and Hom-CKO littermate mice at 2- and 3-weeks-of-age. Mean $\pm \mathrm{SEM}, n=3$ mice/group; ${ }^{*} p<0.05$, one way ANOVA followed by Tukey's modified Student's t-test. Hist1h1c, Histone1, H1c; Hist2h2bb, Histone 2, H2bb.

expression of Klhl31 gene was significantly lower in CSN8CKO hearts when compared to CTL and Het-CKO littermates at 3-weeks-of-age only (Figure 5E). Finally, the expression of a SOCS box containing gene Asb-14 was significantly lower in Hom-CKO hearts at 2- and 3-weeks-of-age. We observed that loss of one CSN8 allele also led to decreased expression of $A s b$-14 gene (Figure 5F). We then decided to look at the protein expression of two SR genes Fbxo31 and Asb-14 in CSN8-CKO heart tissue. FBXO31 protein expression following CSN8-CKO was significantly decreased in Hom-CKO hearts compared to the control group at 2-weeks (Figures 6A,B) and 3-weeks-of-age (Figures 6C,D). Similarly, Asb-14 protein levels in CSN8-CKO mouse hearts were significantly decreased at 2weeks (Figures 7A,B) and 3-weeks-of-age (Figures 7C,D), when compared with control mice.

\section{DEGs Induced by CSN8 Deficiency Enrich in Microtubule-Related Genes}

Since our analyses revealed significant enrichment of DEGs in the microtubule-related pathways in response to CSN8CKO (Supplementary Table 9), we decided to also examine the protein level of differentially expressed microtubule-related genes. Microtubules in the cytoplasm of the cell are involved in vesicular trafficking between endoplasmic reticulum (ER) and Golgi apparatus. Microtubules are also required for chromosome segregation during cell division (Vaughan, 2005). A few studies have reported a link between the CSN-UPS pathway and the microtubule network. For example, failure to degrade MEI1 subunit of the MEI-1/MEI-2 complex that is localized to the spindle and chromosomes during meiosis results in defects in spindle positioning and elongation (Pintard et al., 2003). Another study showed that the downregulation of the CSN led to the reduction of microtubule end-binding protein 1 (EB1) which is a regulator of microtubule dynamics (Peth et al., 2007). CSN3 or CSN5 knockdown caused disrupted meiotic spindle and misarranged chromosomes that result in failure to complete meiosis (Kim et al., 2011). Tubulin tyrosine ligase like-1 (TTLL1) belongs to the family of TTLL enzymes that catalyze the post-translational modifications of tubulin in order to modulate microtubule dynamics and function (Pathak et al., 2011). TTLL1-deficient mice were reported to develop lesions in the respiratory and male reproductive tracts (Vogel et al., 2010). Expression of TTLL1 gene has been found to be significantly decreased in the case of pathological hypertrophy (PAH) (Song et al., 2012). Since previous findings from our lab have reported that CSN8-CKO mice also developed cardiac hypertrophy, we sought to determine the effect of CSN8 deficiency on TTLL1 

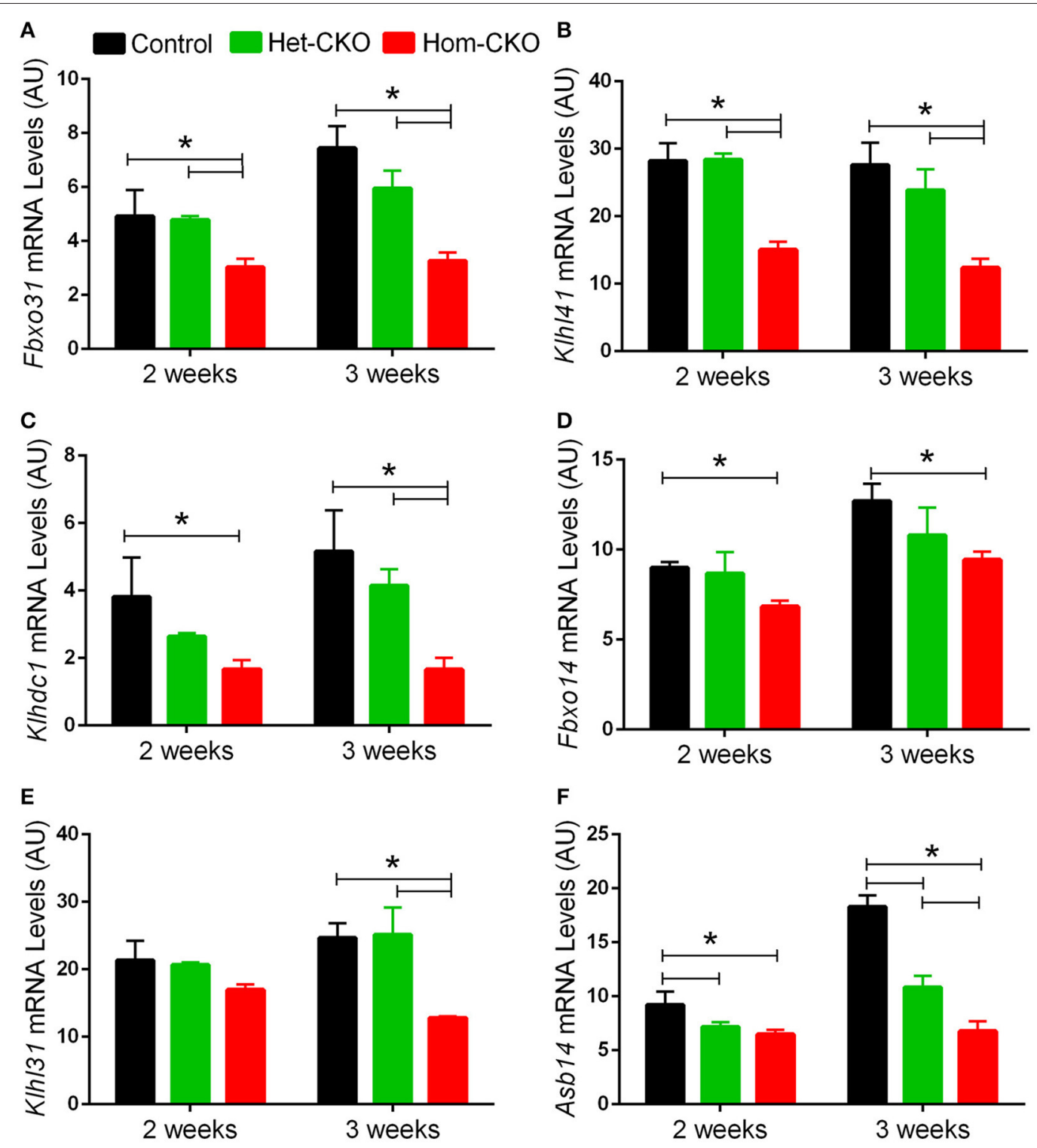

FIGURE 5 | Cardiac CSN8 deficiency causes significant changes in the mRNA levels of myocardial CRL substrate receptors in mice. Shown are microarray-derived mRNA expression levels of the indicated CRL substrate receptors in CTL, Het-CKO, and Hom-CKO littermate mice at 2- and 3-weeks-of-age. (A) Fbxo31, F-box only protein 31; (B) KIhl41, Kelch like 41; (C) KIhdc1, Kelch domain containing 1; (D) Fbxo14, F-box only protein 14; (E) KIh/31, Kelch like 31; (F) Asb14, ankyrin repeat and SOCS box-containing protein 14. Mean \pm SEM, $n=3$ mice/group; * $p<0.05$, one way ANOVA followed by Tukey's modified Student's $t$-test

gene expression. The microarray data revealed that expression of the TTLL1 gene in response to CSN8-CKO was significantly down at both 2 and 3-weeks-of-age (Supplementary Figure 9A). In fact, TTLL1 was among top genes that were downregulated in CSN8-CKO heart tissue (Table 1). The expression of TTLL1 protein in CSN8-CKO heart tissues did not show discernible change at 2-weeks-of-age (Supplementary Figures 9B,C) but it was markedly decreased at 3-weeks-of-age (Supplementary Figures 9D,E), compared with the respective CTL. It will be interesting to determine the role of TTLL1 down-regulation in impaired autophagosome maturation observed in CSN8 deficient cardiomyocytes.

\section{DISCUSSION}

\section{Is CSN8 a Transcriptional Regulator in Murine Hearts?}

Cullin-RING ligases (CRLs) are the largest subfamily of ubiquitin ligases, responsible for approximately $20 \%$ of the ubiquitindependent protein degradation in the cell (Petroski and Deshaies, 2005; Soucy et al., 2009). Although the CSN5-mediated deneddylation of CRLs is the most important activity of the CSN complex, and the loss of this activity results in lethality in most of the cases, not all CRLs are sensitive to deneddylation

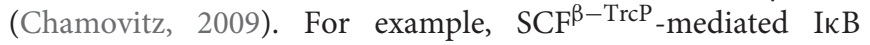



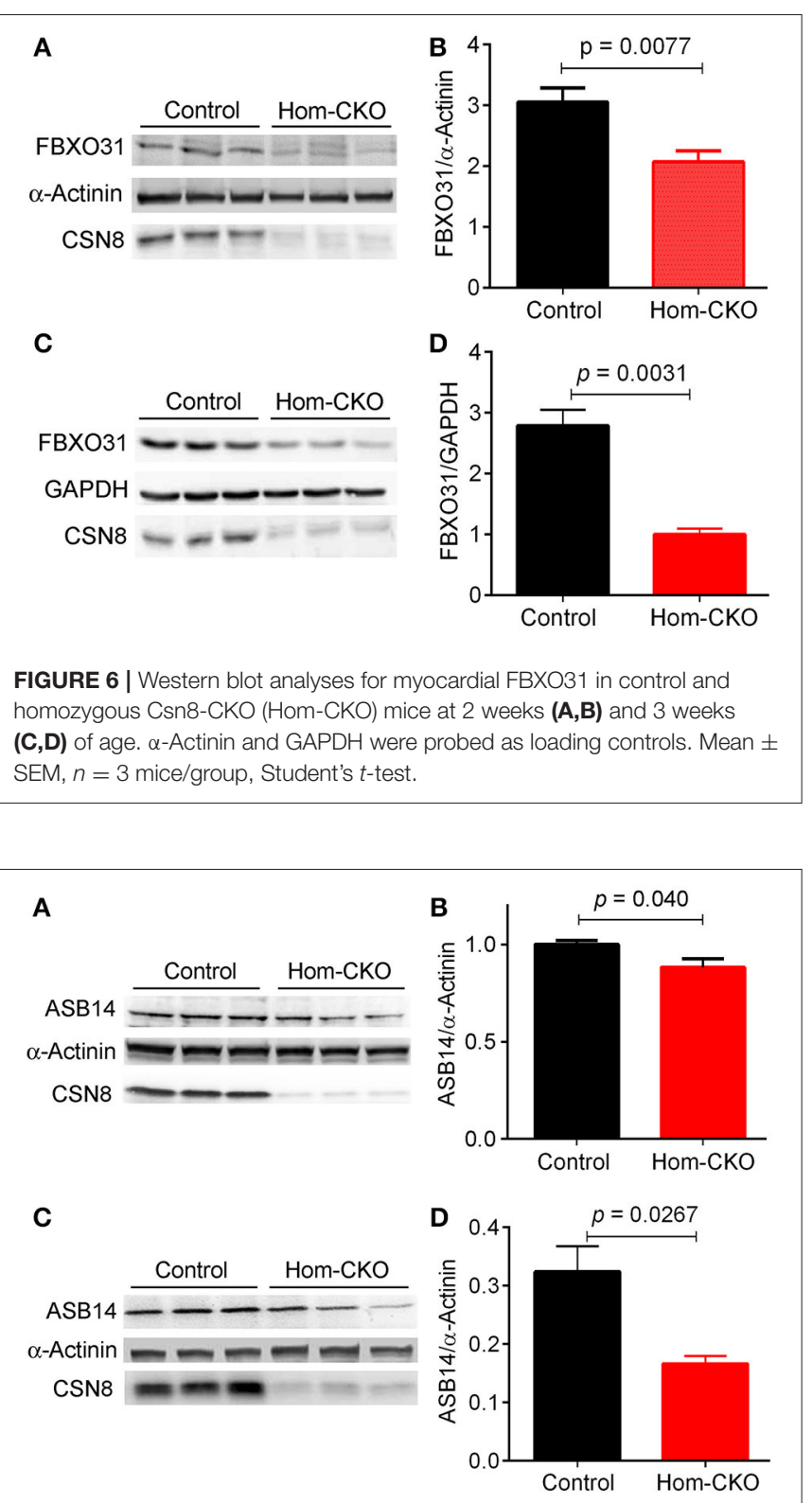

FIGURE 7 | Western blot analyses for myocardial ASB14 in control and homozygous Csn8-CKO (Hom-CKO) mice at 2 weeks $(\mathbf{A}, \mathbf{B})$ and 3 weeks (C,D) of age. $\alpha$-Actinin was probed as loading control. Mean \pm SEM, $n=3$ mice/group, Student's t-test.

degradation, $\mathrm{SCF}^{\mathrm{Skp} 2-}$ mediated downregulation of $\mathrm{p} 27$ and $\mathrm{SCF}^{\text {Slimb }}$-mediated degradation of the IKB homolog Cactus and the clock protein TIM are unaffected after knockdown of different CSN subunits (Harari-Steinberg et al., 2007; Menon et al., 2007; Schweitzer et al., 2007; Panattoni et al., 2008; Knowles et al., 2009). These examples emphasize the importance of CSN's non-deneddylation activities which include deubiquitination, regulation of protein subcellular localization, associated kinase activity and transcription regulation (Chamovitz, 2009). Since the original description of the CSN was given as a repressor of Arabidopsis genes (Wei and Deng, 1992), the role of the
CSN in regulating the activity of transcription factors has been shown in many species (Wei et al., 2008). The mammalian CSN5 was originally identified as a transcriptional co-activator for c-Jun of AP-1 regulatory protein (Claret et al., 1996). In T-lymphocytes, deficiency of CSN8 leads to elevation of cell cycle regulating genes cyclin E1, cyclin D2 and E2F1 relative to wild-type mice (Menon et al., 2007). Our group has also shown previously that down-regulation of CSN8 in HEK293 cells resulted in reduced transcript levels of cyclin kinase inhibitor $\mathrm{p} 21^{\mathrm{cip}}$ and $\mathrm{p} 27^{\mathrm{kip}}$ (Su et al., 2009). In Drosophila, mutation of CSN subunits 4 and 5 leads to dysregulation of the transcriptome during early larval development, which indicates that a primary effect of CSN perturbation is a change in gene expression profiles. Furthermore, these results indicate that in the absence of CSN4 and CSN5, the transcription of numerous early developmental genes is activated. On the other hand, the transcription of a large set of genes was found to be decreased in the absence of the CSN, which also illustrates the CSN as a transcriptional activator (Oron et al., 2007). Our previous studies have identified striking phenotypes in mice with cardiac deficiency of CSN8 (Su et al., 2011a,b); however, the mechanism responsible for CSN8 deficiency to cause the cardiac pathology, such as massive cardiomyocyte necrosis, remains obscure. To explore the potential involvement of altered gene expression, here we performed transcriptome analysis at postnatal 2 and 3 weeks-of-age. Gene microarray data revealed that there were more DEGs in response to CSN8 ablation at 3 weeks than at 2 weeks (Figure 1). In response to CSN8 deficiency, expression of a large number of genes was found to be upregulated, with Prkag3, Cilp, Grm1, Cdkl1, and Parp6 being the top 5 upregulated genes at 2-weeks-of-age (Table 1). On the other hand, we also found a number of genes that showed decreased expression at 2 weeks in response to CSN8-CKO. Among the top down-regulated genes were Syp, Phkg1, Aqp4, Ttll1, and $P f k f b 1$ (Table 2). These results are in agreement with a previous report that the CSN can act as both transcriptional repressor and transcriptional activator (Oron et al., 2007). However, our results are in contrast to earlier description of the CSN as transcriptional repressor only (Wei and Deng, 1992). The potential regulatory role of CSN8 was further evaluated by examining the expression of hand-curated genes of eight different pathways that we expected to be affected by CSN activity (Table 3). The eight different sets of genes include SRs of CRLs and chromatin remodeling genes (discussed in separate sections), autophagy, microtubules, vesicle trafficking, endocytosis, ATP synthases, and those of cell death pathways. All but the cell death pathways were enriched with DEGs. DEGs that show similar expression pattern were clustered together (Figure 2). Moreover, interaction among hand-curated genes is shown in co-expression network map (Supplementary Figure 8). This network shows changes in interaction of a single gene following CSN8-CKO. Synapsin II (vesicle trafficking) and TTLL1 (microtubules) were among top five DEGs in response to CSN8 ablation in mouse hearts (Tables 1, 2). Further investigation reveals that TTLL1 protein levels were significantly decreased in Hom-CKO mouse hearts at 3 weeks of age (Supplementary Figure 9). Together these data are consistent with the notion that CSN8/CSN likely 
regulates the gene expression of multiple pathways in murine hearts.

\section{Is CSN8 a Chromatin-Based Transcriptional Regulator?}

Several studies have suggested the involvement of the CSN in transcriptional regulation and that the CSN regulates transcription either by regulating the protein stability of transcription factors or by directly acting on chromatin along with other components of the UPS (Chamovitz, 2009). Various transcription factors have been identified as interactors of CSN subunits (Kato and Yoneda-Kato, 2009). Drosophila CSN4 was reported to interact with Rbf-targeted promotor region of Pol $\alpha$ and PCNA genes after targeting Rbf protein (Ullah et al., 2007). CSN1 and CSN8 were reported to bind to Ccnd2, Cdk4 and Cdknla promotors (Menon et al., 2007). CSN5 was originally identified as a coactivator of Ap-1 transcription factor binding sites (Claret et al., 1996). CSN2 was originally identified as a corepressor of steroid hormone signaling (Dressel et al., 1999). To our knowledge, a direct effect of CSN subunits on chromatin has not been reported. Here we have shown for the first time that CSN8 deficiency leads to decreased expression of two of the histone genes; Hist $1 h 1 c$ and Hist2h2bb (Figure 4). These two, along with other histone proteins, are responsible for keeping the fidelity and regulating access to genomic DNA in order to modulate gene transcription (Hughes and Rando, 2014). Hence, it is tempting to hypothesize that CSN8 may regulate gene transcription through chromatin remodeling. To test this hypothesis we will need to determine whether the decreased mRNA levels are accompanied by altered protein levels and examine the interaction of CSN8 with the regulatory elements of these histone genes.

\section{CSN8 May Regulate the Transcription of CRL Substrate Receptors}

The specificity of CRLs toward a target protein depends on the SR module binding to the amino terminus of cullin scaffold (Duda et al., 2011). It has been estimated that the human genome encodes about 200 SR's, each of which may bind with one of the eight cullins to form a specific CRL (Wang and Martin, 2015). Members of CRLs and more specifically, their SRs, play important roles in numerous physiological functions and dysfunction and thereby are involved in a wide range of diseases (Bulatov and Ciulli, 2015). Several SRs, for example, members of the F-box protein family, such as Skp2, $\beta$-TrCP, Fbw7 and Fbxl3, were shown to play significant roles in cancer and other diseases (Wang et al., 2014). Overexpression of $\beta$ $\mathrm{TrCP}$ has been reported in different types of cancer and during the inflammatory process (Frescas and Pagano, 2008; Skaar et al., 2013). Another SR VHL recognizes hypoxia inducible factor $(\mathrm{HIF} \alpha)$ for degradation by CRL2 ${ }^{\mathrm{VHL}}$ and acts as a tumor suppressor protein. Mutation of VHL is reported in von HippelLindau (VHL) hereditary cancer syndrome (Zhang and Yang, 2012). Similarly, Fem 1 b of CRL2 ${ }^{\text {FEMB1 }}$ promotes ubiquitination and suppressed transcriptional activity of Gli1 oncoprotein in humans (Gilder et al., 2013). It is for these reasons that the stability of SRs of CRLs needs to be regulated efficiently. The detachment of NEDD8 from activated CRLs is believed to prevent autoubiquitination and subsequent destruction of the SR when the substrate is running low. This is also necessary for the disassembly and remodeling of CRL complexes (Duda et al., 2011; Pierce et al., 2013; Wang and Martin, 2015). Although cells and tissues deficient of CSN subunits showed decreased protein levels of SRs (Wee et al., 2005; Cope and Deshaies, 2006; Su et al., 2011b) it has not yet been confirmed if the known reduction of SR protein levels can be caused by decreased expression at the transcription level, in addition to the proposed self-destruction of the SR proteins. Our results have clearly shown that CSN8 deficiency leads to decreases in both the mRNA levels (Figure 5, Supplementary Tables 3,4) and the protein levels (Figures 6, 7) of a number of SRs of the F-box and SOCS-box families. To our best knowledge, this is the first study that has reported the transcript levels of SR genes are markedly down-regulated by ablation of a CSN subunit. In general, a decrease in the protein level of a gene is expected to upregulate its transcription via a negative feedback mechanism. As exemplified by FBXO31 and ASB14, we observed concomitant decreases in both the mRNA levels and the protein levels for SRs in Hom-CKO mouse hearts at both 2 and 3 weeks of age, suggesting that the decreased transcription contributes to the decreased protein levels. Autoubiquitination and resultant self-destruction of the SR in the CRL has been the predominant theory for explaining the decreased SR proteins observed in CSN deficient cells and, based on this theory, the mechanism by which the CSN regulates a CRL has been commonly believed to preserve its own SR upon the completion of ubiquitination of its substrates by the CRL. Our findings reported here obviously challenge this theory. Hence, we propose that CSN8/CSN regulates the SR protein levels and thereby the activity of CRLs through both transcriptional and post-translational mechanisms.

\section{AUTHOR CONTRIBUTIONS}

XW, Conception and experimental design of the study, data analysis and interpretation, and manuscript preparation; AA, Data collection, analysis, interpretation, and manuscript preparation; KE, Experimental design, data collection and analyses, and preparation of the manuscript; TB, PX data collection and analyses; EZ, bioinformatics and statistical analyses of the microarray data, preparation of the related sections of the manuscript.

\section{FUNDING}

This work is supported in part by National Institutes of Health grants HL072166, HL085629, and HL131667. The Genomics Core facility at University of South Dakota is supported by NIH P20 GM103443.

\section{SUPPLEMENTARY MATERIAL}

The Supplementary Material for this article can be found online at: http://journal.frontiersin.org/article/10.3389/fphys. 2017.00594/full\#supplementary-material 


\section{REFERENCES}

Amare, A. T., Schubert, K. O., Klingler-Hoffmann, M., Cohen-Woods, S., and Baune, B. T. (2017). The genetic overlap between mood disorders and cardiometabolic diseases: a systematic review of genome wide and candidate gene studies. Transl. Psychiatry 7:e1007. doi: 10.1038/tp.2016.261

Bezniakow, N., Gos, M., and Obersztyn, E. (2014). The RASopathies as an example of RAS/MAPK pathway disturbances-clinical presentation and molecular pathogenesis of selected syndromes. Dev. Period Med. 18, 285-296.

Bosu, D. R., and Kipreos, E. T. (2008). Cullin-RING ubiquitin ligases: global regulation and activation cycles. Cell Div. 3:7. doi: 10.1186/1747-1028-3-7

Brazma, A., Hingamp, P., Quackenbush, J., Sherlock, G., Spellman, P., Stoeckert, C., et al. (2001). Minimum information about a microarray experiment (MIAME)-toward standards for microarray data. Nat. Genet. 29, 365-371. doi: 10.1038/ng1201-365

Bulatov, E., and Ciulli, A. (2015). Targeting Cullin-RING E3 ubiquitin ligases for drug discovery: structure, assembly and small-molecule modulation. Biochem. J. 467, 365-386. doi: 10.1042/BJ20141450

Burnstock, G. (2017). Purinergic signaling in the cardiovascular system. Circ. Res. 120, 207-228. doi: 10.1161/CIRCRESAHA.116.309726

Busch, S., Schwier, E. U., Nahlik, K., Bayram, O., Helmstaedt, K., Draht, O. W., et al. (2007). An eight-subunit COP9 signalosome with an intact JAMM motif is required for fungal fruit body formation. Proc. Natl. Acad. Sci. U.S.A. 104, 8089-8094. doi: 10.1073/pnas.0702108104

Chamovitz, D. A. (2009). Revisiting the COP9 signalosome as a transcriptional regulator. $E M B O$ Rep. 10, 352-358. doi: 10.1038/embor.2009.33

Claret, F. X., Hibi, M., Dhut, S., Toda, T., and Karin, M. (1996). A new group of conserved coactivators that increase the specificity of AP-1 transcription factors. Nature 383, 453-457. doi: 10.1038/383453a0

Cope, G. A., and Deshaies, R. J. (2006). Targeted silencing of Jab1/Csn5 in human cells downregulates SCF activity through reduction of F-box protein levels. BMC Biochem. 7:1. doi: 10.1186/1471-2091-7-1

Cope, G. A., Suh, G. S. B., Aravind, L., Schwarz, S. E., Zipursky, S. L., Koonin, E. V., et al. (2002). Role of predicted metalloprotease motif of jab1/Csn5 in cleavage of nedd8 from Cul1. Science 298, 608-611. doi: 10.1126/science.1075901

Dietrich, C. (2016). Antioxidant functions of the Aryl hydrocarbon RECEPTOR. Stem Cells Int. 2016:7943495. doi: 10.1155/2016/7943495

Dressel, U., Thormeyer, D., Altincicek, B., Paululat, A., Eggert, M., Schneider, S., et al. (1999). Alien, a highly conserved protein with characteristics of a corepressor for members of the nuclear hormone receptor superfamily. Mol. Cell. Biol. 19, 3383-3394. doi: 10.1128/MCB.19.5.3383

Duda, D. M., Scott, D. C., Calabrese, M. F., Zimmerman, E. S., Zheng, N., and Schulman, B. A. (2011). Structural regulation of cullin-RING ubiquitin ligase complexes. Curr. Opin. Struct. Biol. 21, 257-264. doi: 10.1016/j.sbi.2011.01.003

Emberley, E. D., Mosadeghi, R., and Deshaies, R. J. (2012). Deconjugation of Nedd8 from Cul1 is directly regulated by Skp1-F-box and substrate, and the COP9 signalosome inhibits deneddylated SCF by a noncatalytic mechanism. J. Biol. Chem. 287, 29679-29689. doi: 10.1074/jbc.M112.352484

Eyster, K. M., Klinkova, O., Kennedy, V., and Hansen, K. A. (2007). Whole genome deoxyribonucleic acid microarray analysis of gene expression in ectopic versus eutopic endometrium. Fertil. Steril. 88, 1505-1533. doi: 10.1016/j.fertnstert.2007.01.056

Fabiato, A. (1992). Two kinds of calcium-induced release of calcium from the sarcoplasmic reticulum of skinned cardiac cells. Adv. Exp. Med. Biol. 311, 245-262. doi: 10.1007/978-1-4615-3362-7_18

Frescas, D., and Pagano, M. (2008). Deregulated proteolysis by the F-box proteins SKP2 and beta-TrCP: tipping the scales of cancer. Nat. Rev. Cancer 8, 438-449. doi: $10.1038 / \mathrm{nrc} 2396$

Gilder, A. S., Chen, Y. B., Jackson, R. J. III.. Jiang, J., and Maher, J. F. (2013). Fem $1 \mathrm{~b}$ promotes ubiquitylation and suppresses transcriptional activity of Gli1. Biochem. Biophys. Res. Commun. 440, 431-436. doi: 10.1016/j.bbrc.2013.09.090

Harari-Steinberg, O., Cantera, R., Denti, S., Bianchi, E., Oron, E., Segal, D., et al. (2007). COP9 signalosome subunit 5 (CSN5/Jab1) regulates the development of the Drosophila immune system: effects on Cactus, Dorsal and hematopoiesis. Genes Cells 12, 183-195. doi: 10.1111/j.1365-2443.2007.01049.x

Hughes, A. L., and Rando, O. J. (2014). Mechanisms underlying nucleosome positioning in vivo. Annu. Rev. Biophys. 43, 41-63. doi: 10.1146/annurev-biophys-051013-023114
Kalil, A. C., and Florescu, D. F. (2013). Severe sepsis: are PROWESS and PROWESS-SHOCK trials comparable? A clinical and statistical heterogeneity analysis. Crit Care 17:167. doi: 10.1186/cc12752

Kato, J. Y., and Yoneda-Kato, N. (2009). Mammalian COP9 signalosome. Genes Cells 14, 1209-1225. doi: 10.1111/j.1365-2443.2009.01349.x

Kim, E., Yoon, S. J., Kim, E. Y., Kim, Y., Lee, H. S., Kim, K. H., et al. (2011). Function of COP9 signalosome in regulation of mouse oocytes meiosis by regulating MPF activity and securing degradation. PLOS ONE 6:e25870. doi: 10.1371/journal.pone.0025870

Knowles, A., Koh, K., Wu, J. T., Chien, C. T., Chamovitz, D. A., and Blau, J. (2009). The COP9 signalosome is required for light-dependent timeless degradation and Drosophila clock resetting. J. Neurosci. 29, 1152-1162. doi: 10.1523/JNEUROSCI.0429-08.2009

Lei, D., Li, F., Su, H., Liu, J., Wei, N., and Wang, X. (2013). Hepatic deficiency of COP9 signalosome subunit 8 induces ubiquitin-proteasome system impairment and Bim-mediated apoptosis in murine livers. PLoS ONE 8:e67793. doi: 10.1371/journal.pone.0067793

Lei, D., Li, F., Su, H., Tian, Z., Ye, B., Wei, N., et al. (2011). COP9 signalosome subunit 8 is required for postnatal hepatocyte survival and effective proliferation. Cell Death Differ. 18, 259-270. doi: 10.1038/cdd.2010.98

Lingaraju, G. M., Bunker, R. D., Cavadini, S., Hess, D., Hassiepen, U., Renatus, M., et al. (2014). Crystal structure of the human COP9 signalosome. Nature 512, 161-165. doi: 10.1038/nature13566

Lyapina, S., Cope, G., Shevchenko, A., Serino, G., Tsuge, T., Zhou, C., et al. (2001). Promotion of NEDD8-CUL1 Conjugate Cleavage by COP9 Signalosome. Science 292, 1382-1385. doi: 10.1126/science. 1059780

Menon, S., Chi, H., Zhang, H., Deng, X. W., Flavell, R. A., and Wei, N. (2007) COP9 signalosome subunit 8 is essential for peripheral $\mathrm{T}$ cell homeostasis and antigen receptor-induced entry into the cell cycle from quiescence. Nat. Immunol. 8, 1236-1245. doi: 10.1038/ni1514

Moss, R. L., and Fitzsimons, D. P. (2006). Myosin light chain 2 into the mainstream of cardiac development and contractility. Circ. Res. 99, 225-227. doi: 10.1161/01.RES.0000236793.88131.dc

Murthy, K. S. (2006). Signaling for contraction and relaxation in smooth muscle of the gut. Annu. Rev. Physiol. 68, 345-374. doi: 10.1146/annurev.physiol.68.040504.094707

Oron, E., Tuller, T., Li, L., Rozovsky, N., Yekutieli, D., Rencus-Lazar, S., et al. (2007). Genomic analysis of COP9 signalosome function in Drosophila melanogaster reveals a role in temporal regulation of gene expression. Mol. Syst. Biol. 3:108. doi: 10.1038/msb4100150

Panattoni, M., Sanvito, F., Basso, V., Doglioni, C., Casorati, G., Montini, E., et al. (2008). Targeted inactivation of the COP9 signalosome impairs multiple stages of T cell development. J. Exp. Med. 205, 465-477. doi: 10.1084/jem.20070725

Pathak, N., Austin, C. A., and Drummond, I. A. (2011). Tubulin tyrosine ligaselike genes ttll3 and ttll6 maintain zebrafish cilia structure and motility. J. Biol. Chem. 286, 11685-11695. doi: 10.1074/jbc.M110.209817

Peth, A., Boettcher, J. P., and Dubiel, W. (2007). Ubiquitin-dependent proteolysis of the microtubule end-binding protein $1, \mathrm{~EB} 1$, is controlled by the COP9 signalosome: possible consequences for microtubule filament stability. J. Mol. Biol. 368, 550-563. doi: 10.1016/j.jmb.2007.02.052

Petroski, M. D., and Deshaies, R. J. (2005). Function and regulation of cullin-RING ubiquitin ligases. Nat. Rev. Mol. Cell Biol. 6, 9-20. doi: 10.1038/nrm1547

Pierce, N. W., Lee, J. E., Liu, X., Sweredoski, M. J., Graham, R. L., Larimore, E. A., et al. (2013). Cand1 promotes assembly of new SCF complexes through dynamic exchange of F box proteins. Cell 153, 206-215. doi: 10.1016/j.cell.2013.02.024

Pintard, L., Kurz, T., Glaser, S., Willis, J. H., Peter, M., and Bowerman, B. (2003). Neddylation and deneddylation of CUL-3 is required to target MEI-1/Katanin for degradation at the meiosis-to-mitosis transition in C. elegans. Curr. Biol. 13, 911-921. doi: 10.1016/S0960-9822(03)00336-1

Ponicke, K., Giessler, C., Grapow, M., Heinroth-Hoffmann, I., Becker, K., Osten, B., et al. (2000). FP-receptor mediated trophic effects of prostanoids in rat ventricular cardiomyocytes. Br. J. Pharmacol. 129, 1723-1731. doi: 10.1038/sj.bjp. 0703243

Pugach, E. K., Richmond, P. A., Azofeifa, J. G., Dowell, R. D., and Leinwand, L. A. (2015). Prolonged Cre expression driven by the alpha-myosin heavy chain promoter can be cardiotoxic. J. Mol. Cell. Cardiol. 86, 54-61. doi: 10.1016/j.yjmcc.2015.06.019 
Rose, B. A., Force, T., and Wang, Y. (2010). Mitogen-activated protein kinase signaling in the heart: angels versus demons in a heart-breaking tale. Physiol. Rev. 90, 1507-1546. doi: 10.1152/physrev.00054.2009

Schlierf, A., Altmann, E., Quancard, J., Jefferson, A. B., Assenberg, R., Renatus, M., et al. (2016). Targeted inhibition of the COP9 signalosome for treatment of cancer. Nat. Commun. 7:13166. doi: 10.1038/ncomms13166

Schmidt, M. W., McQuary, P. R., Wee, S., Hofmann, K., and Wolf, D. A. (2009). Fbox-directed CRL complex assembly and regulation by the CSN and CAND1. Mol. Cell 35, 586-597. doi: 10.1016/j.molcel.2009.07.024

Schweitzer, K., Bozko, P. M., Dubiel, W., and Naumann, M. (2007). CSN controls NF-kappaB by deubiquitinylation of IkappaBalpha. EMBO J. 26, 1532-1541. doi: 10.1038/sj.emboj.7601600

Skaar, J. R., Pagan, J. K., and Pagano, M. (2013). Mechanisms and function of substrate recruitment by F-box proteins. Nat. Rev. Mol. Cell Biol. 14, 369-381. doi: $10.1038 / \mathrm{nrm} 3582$

Song, H. K., Hong, S. E., Kim, T., and Kim, D. H. (2012). Deep RNA sequencing reveals novel cardiac transcriptomic signatures for physiological and pathological hypertrophy. PLoS ONE 7:e35552. doi: 10.1371/journal.pone.0035552

Soucy, T. A., Smith, P. G., Milhollen, M. A., Berger, A. J., Gavin, J. M., Adhikari, S., et al. (2009). An inhibitor of NEDD8-activating enzyme as a new approach to treat cancer. Nature 458, 732-736. doi: 10.1038/nature07884

Sozen, E., Karademir, B., and Ozer, N. K. (2015). Basic mechanisms in endoplasmic reticulum stress and relation to cardiovascular diseases. Free Radic. Biol. Med. 78, 30-41. doi: 10.1016/j.freeradbiomed.2014.09.031

Stelzer, J. E., Patel, J. R., and Moss, R. L. (2006). Acceleration of stretch activation in murine myocardium due to phosphorylation of myosin regulatory light chain. J. Gen. Physiol. 128, 261-272. doi: 10.1085/jgp.200609547

Su, H., Huang, W., and Wang, X. (2009). The COP9 signalosome negatively regulates proteasome proteolytic function and is essential to transcription. Int. J. Biochem. Cell Biol. 41, 615-624. doi: 10.1016/j.biocel.2008.07.008

Su, H., Li, F., Ranek, M. J., Wei, N., and Wang, X. (2011a). COP9 signalosome regulates autophagosome maturation. Circulation 124, 2117-2128. doi: 10.1161/CIRCULATIONAHA.111.048934

Su, H., Li, J., Menon, S., Liu, J., Kumarapeli, A. R., Wei, N., et al. (2011b). Perturbation of cullin deneddylation via conditional Csn8 ablation impairs the ubiquitin-proteasome system and causes cardiomyocyte necrosis and dilated cardiomyopathy in mice. Circ. Res. 108, 40-50. doi: 10.1161/CIRCRESAHA.110.230607

Su, H., Li, J., Osinska, H., Li, F., Robbins, J., Liu, J., et al. (2013). The COP9 signalosome is required for autophagy, proteasome-mediated proteolysis, and cardiomyocyte survival in adult mice. Circ. Heart Fail. 6, 1049-1057. doi: 10.1161/CIRCHEARTFAILURE.113.000338

Subedi, K. P., Son, M. J., Chidipi, B., Kim, S. W., Wang, J., Kim, K. H., et al. (2017). Signaling pathway for endothelin-1- and phenylephrineinduced cAMP response element binding protein activation in rat ventricular myocytes: role of inositol 1,4,5-Trisphosphate Receptors and CaMKII. Cell. Physiol. Biochem. 41, 399-412. doi: 10.1159/0004 56422

Takayama, K., Yuhki, K., Ono, K., Fujino, T., Hara, A., Yamada, T., et al. (2005). Thromboxane $\mathrm{A}_{2}$ and prostaglandin $\mathrm{F}_{2} \alpha$ mediate inflammatory tachycardia. Nat. Med. 11, 562-566. doi: 10.1038/nm1231
Tracey, K. J. (2002). The inflammatory reflex. Nature 420, 853-859. doi: 10.1038 /nature 01321

Ullah, Z., Buckley, M. S., Arnosti, D. N., and Henry, R. W. (2007). Retinoblastoma protein regulation by the COP9 signalosome. Mol. Biol. Cell 18, 1179-1186. doi: $10.1091 / \mathrm{mbc}$.E06-09-0790

Valen, G. (2011). Innate immunity and remodelling. Heart Fail. Rev. 16, 71-78. doi: 10.1007/s10741-010-9187-1

Vaughan, K. T. (2005). Microtubule plus ends, motors, and traffic of Golgi membranes. Biochim. Biophys. Acta 1744, 316-324. doi: 10.1016/j.bbamcr.2005.05.001

Vogel, P., Hansen, G., Fontenot, G., and Read, R. (2010). Tubulin tyrosine ligase-like 1 deficiency results in chronic rhinosinusitis and abnormal development of spermatid flagella in mice. Vet. Pathol. 47, 703-712. doi: $10.1177 / 0300985810363485$

Wang, X., and Martin, D. S. (2015). The COP9 signalosome and cullin-RING ligases in the heart. Am. J. Cardiovasc. Dis. 5, 1-18.

Wang, Z., Liu, P., Inuzuka, H., and Wei, W. (2014). Roles of F-box proteins in cancer. Nat. Rev. Cancer 14, 233-247. doi: 10.1038/nrc3700

Wee, S., Geyer, R. K., Toda, T., and Wolf, D. A. (2005). CSN facilitates Cullin-RING ubiquitin ligase function by counteracting autocatalytic adapter instability. Nat. Cell Biol. 7, 387-391. doi: 10.1038/ncb1241

Wei, N., and Deng, X. W. (1992). COP9: a new genetic locus involved in light-regulated development and gene expression in Arabidopsis. Plant Cell 4, 1507-1518. doi: 10.1105/tpc.4.12.1507

Wei, N., and Deng, X. W. (2003). The COP9 signalosome. Annu. Rev. Cell Dev. Biol. 19, 261-286. doi: 10.1146/annurev.cellbio.19.111301.112449

Wei, N., and Deng, X.-W. (1999). Making sense of the COP9 signalosome: a regulatory protein complex conserved from Arabidopsis to human. Trends Genet. 15, 98-103. doi: 10.1016/S0168-9525(98)01670-9

Wei, N., Chamovitz, D. A., and Deng, X. W. (1994). Arabidopsis COP9 is a component of a novel signaling complex mediating light control of development. Cell 78, 117-124. doi: 10.1016/0092-8674(94)90578-9

Wei, N., Serino, G., and Deng, X. W. (2008). The COP9 signalosome: more than a protease. Trends Biochem. Sci. 33, 592-600. doi: 10.1016/j.tibs.2008.09.004

Zhang, Q., and Yang, H. (2012). The roles of VHL-dependent ubiquitination in signaling and cancer. Front. Oncol. 2:35. doi: 10.3389/fonc.2012.00035

Zhang, Z., Murtagh, F., Van Poucke, S., Lin, S., and Lan, P. (2017). Hierarchical cluster analysis in clinical research with heterogeneous study population: highlighting its visualization with R. Ann. Transl. Med. 5, 75. doi: $10.21037 /$ atm.2017.02.05

Conflict of Interest Statement: The authors declare that the research was conducted in the absence of any commercial or financial relationships that could be construed as a potential conflict of interest.

Copyright (C) 2017 Abdullah, Eyster, Bjordahl, Xiao, Zeng and Wang. This is an open-access article distributed under the terms of the Creative Commons Attribution License (CC BY). The use, distribution or reproduction in other forums is permitted, provided the original author(s) or licensor are credited and that the original publication in this journal is cited, in accordance with accepted academic practice. No use, distribution or reproduction is permitted which does not comply with these terms. 